\title{
Tests with a Carlina-type diluted telescope Primary coherencing
}

\author{
H. Le Coroller ${ }^{1}$, J. Dejonghe ${ }^{1}$, X. Regal ${ }^{1}$, R. Sottile ${ }^{1}$, D. Mourard ${ }^{2}$, D. Ricci ${ }^{3, \star}$, O. Lardiere ${ }^{4}$, A. Le Vansuu ${ }^{1}$, \\ M. Boer ${ }^{1}$, M. De Becker ${ }^{3}$, J.-M. Clausse ${ }^{2}$, C. Guillaume ${ }^{1}$, and J. P. Meunier ${ }^{1}$ \\ ${ }^{1}$ Observatoire de Haute-Provence, 04870 Saint-Michel l'Observatoire, France \\ e-mail: [herve.lecoroller; julien.dejonghe]@oamp.fr \\ 2 Université Nice-Sophia Antipolis, Observatoire de la Côte d'Azur, CNRS UMR 6525, BP 4229, 06304 Nice Cedex, France \\ ${ }^{3}$ Département d'Astrophysique, Géophysique et Océanographie, Bât. B5C, Sart Tilman, Université de Liège, 4000 Liège 1, Belgium \\ 4 AO Laboratory, Mechanical Engineering Department, University of Victoria, PO Box 3055 STN CSC, Victoria, BC, V8W 3P6, \\ Canada
}

Received 24 June 2011 / Accepted 14 December 2011

\section{ABSTRACT}

\begin{abstract}
Aims. Studies are under way to propose a new generation of post-VLTI interferometers. The Carlina concept studied at the HauteProvence Observatory is one of the proposed solutions. It consists in an optical interferometer configured like a diluted version of the Arecibo radio telescope: above the diluted primary mirror made of fixed cospherical segments, a helium balloon (or cables suspended between two mountains), carries a gondola containing the focal optics. Since 2003, we have been building a technical demonstrator of this diluted telescope. First fringes were obtained in May 2004 with two closely-spaced primary segments and a CCD on the focal gondola. We have been testing the whole optical train with three primary mirrors. The main aim of this article is to describe the metrology that we have conceived, and tested under the helium balloon to align the primary mirrors separate by $5-10 \mathrm{~m}$ on the ground with an accuracy of a few microns.

Methods. Getting stellar fringes using delay lines is the main difficulty for astronomical interferometers. Carlina does not use delay lines, but the primary segments have to be positioned on a sphere i.e. coherencing the primary mirrors. As described in this paper, we used a supercontinuum laser source to coherence the primary segments. We characterize the Carlina's performances by testing its whole optical train: servo loop, metrology, and the focal gondola.

Results. The servo loop stabilizes the mirror of metrology under the helium balloon with an accuracy better than $5 \mathrm{~mm}$ while it moves horizontally by $30 \mathrm{~cm}$ in open loop by $10-20 \mathrm{~km} / \mathrm{h}$ of wind. We have obtained the white fringes of metrology; i.e., the three mirrors are aligned (cospherized) with an accuracy of $\approx 1 \mu \mathrm{m}$. We show data proving the stability of fringes over 15 min, therefore providing evidence that the mechanical parts are stabilized within a few microns. This is an important step that demonstrates the feasibility of building a diluted telescope using cables strained between cliffs or under a balloon. Carlina, like the MMT or LBT, could be one of the first members of a new class of telescopes named diluted telescopes.
\end{abstract}

Key words. instrumentation: interferometers - atmospheric effects - instrumentation: adaptive optics - telescopes - balloons instrumentation: high angular resolution

\section{Introduction}

Studies are under way to propose a new generation of postVLTI interferometers. For example, the OHANA team has proposed to recombine distant telescopes with optical fibers (Perrin et al. 2000; Woillez et al. 2005). Other teams work on new types of combiners that could provide direct snapshot images, and increase the sensitivity of numerous aperture interferometers (Tallon 1992; Labeyrie 1996; Lardière et al. 2003; Patru et al. 2009). The post-VLTI area will be driven by new fields of research in astrophysics, such as stellar surface imaging, studies of gravitational microlensing and central torus of AGN, and exoplanets. To achieve these goals, they will have to meet several criteria (Ridgway \& Glindemann 2010): high angular resolution (Baseline $>100 \mathrm{~m}$ ), a good coverage of uv spatial frequencies (large number of mirrors), and better sensitivity than regular interferometers (VLTI, Keck, CHARA, etc.). They will be able to accomodate various focal instruments, such as spectrographs or

\footnotetext{
* FRIA fellow.
}

coronagraphs. A diluted telescope like Carlina could meet all these criteria.

The optomechanical design of Carlina was described in Le Coroller et al. (2004, hereafter Paper I). It is similar to the Arecibo radio telescope, but its spherical primary mirror is diluted (Fig. 3 in Paper I) and operates at visible wavelengths. There are no delay lines, and it can work with hundreds of mirrors and should be very efficient thanks to the few intermediate optical surfaces between the primary mirrors and the final detector. The stability of the mirrors anchored in the bedrock and an internal metrology system described in this paper also offer strong advantages.

The star light is focalized on the focal gondola (at $R / 2$ ) that contains the Mertz (1996) sphericity corrector, a tracking system, a pupil densifier (Tallon 1992; Labeyrie 1996), and a focal instrument such as a photon counting camera (Blazit et al. 2008). We will describe this complex focal optical and mechanical set up, its implementation, and the first observations in a forthcoming paper. In this article, we deal with the metrology at 


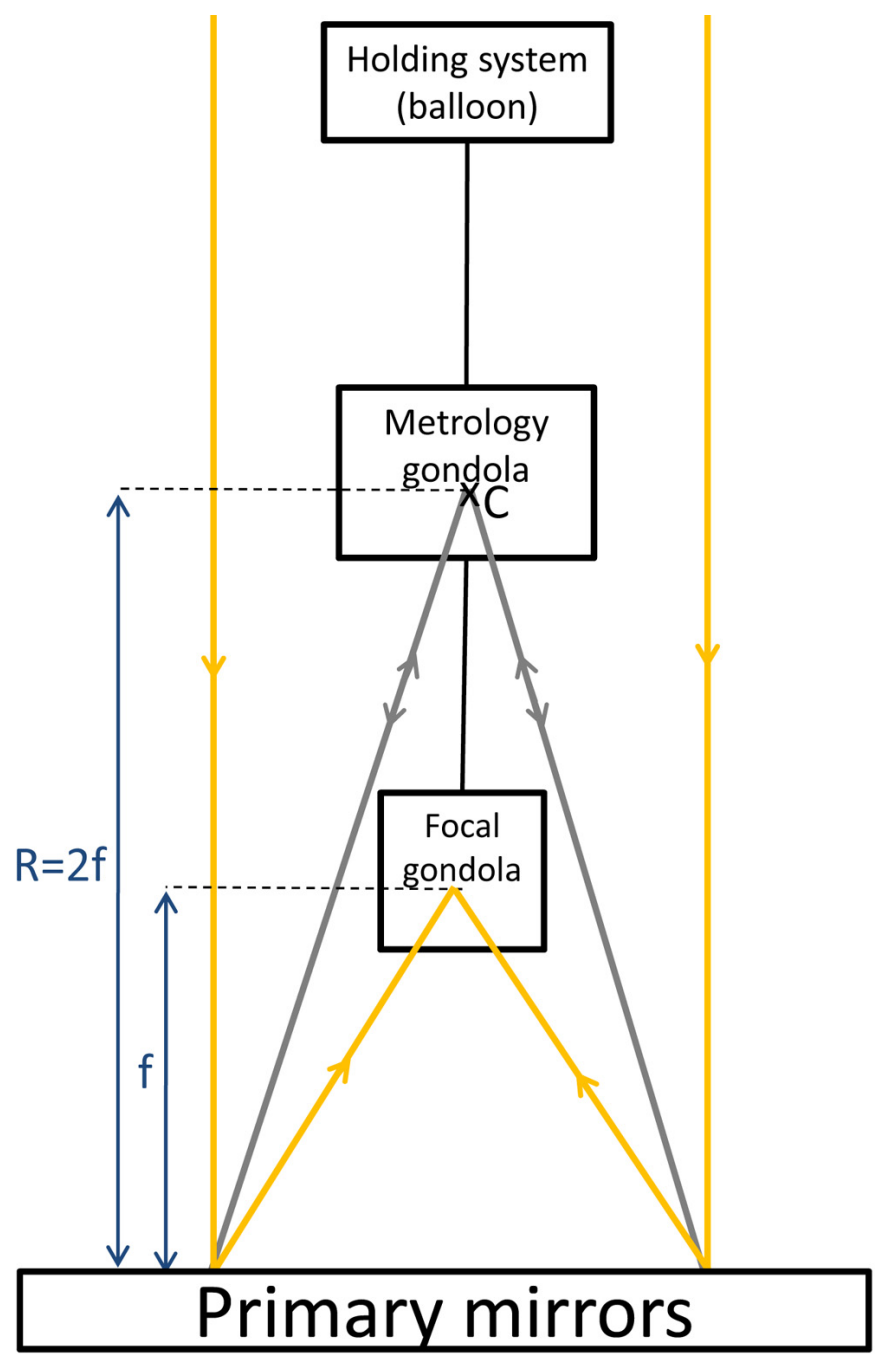

Fig. 1. Block diagram of the Carlina project. It is divided in 4 blocks: the primary mirrors, the focal gondola, the metrology gondola, and the holding system that carry the gondolas. The yellow lines show the stellar light, and the gray lines the coherencing laser beam.

the curvature center of the diluted primary mirror to align (coherencing) the primary mirrors.

In Sect. 2, we present the goal of the OHP prototype and the specifications for such a project. In Sect. 3, we briefly recall the general principles of Carlina. In Sect. 4, we describe the optical and mechanical solutions that we conceived to build a prototype that responds to the required specifications. The alignment procedure is provided in Sect. 5. Results are presented at Sect. 6. We conclude in Sect. 7 and propose the idea of a $100 \mathrm{~m}$ aperture scientific demonstrator named the large diluted telescope (LDT). A diluted telescope working with hundreds of mirrors could complement ELTs (D'Odorico 2010) and kilometer interferometers (Meisenheimer 2009).

\section{Experiment goals and specifications}

The general architecture of Carlina has been detailed in Paper I. This diluted telescope can be divided in four parts linked with each other as described in the block diagram at Fig. 1 and in Sect. 3. These four parts are:

- a spherical diluted primary mirror made of numerous small mirrors;
- a focal gondola that tracks the stars by moving on the focal sphere at the half radius of the spherical diluted primary mirror;

- a metrology gondola at the curvature center of the diluted primary mirror. It is used to adjust the mirrors position on the primary sphere with high accuracy (primary coherencing). A part of this metrology gondola (source, and CCD) can be placed on the ground (Sect. 4.1.2);

- a holding system that carries the metrology and focal gondola.

The main goal of the experiment described in this paper is to obtain stable metrology fringes in order to align the primary mirrors with one micron accuracy i.e. we have to obtain fringes that stay in the field of view of the metrology CCD and that move slowly enough to be frozen in a short time exposure $(\approx 1 \mathrm{~ms})$. We provide the specifications in order to reach this goal in Appendix A. In the next sections, we describe in detail the Carlina architecture and the solutions found with the OHP prototype in order to answer to the specifications of Table A.1. We will see that the OHP prototype reaches perfectly these specifications (last column of Table A.1). The focal gondola characteristics will however be described in a forthcoming paper.

\section{General principles}

A cable tripod tensioned by a helium balloon, or cables attached between two mountains ensures that the suspended optics is stabilized within the limits defined by the cable sag. The top of this tripod is also the curvature center of the spherical diluted primary mirror (Fig. 3 in Paper I). This point is used for the alignment of the primary segments using a metrology gondola. Under the metrology gondola, a focal gondola is constrained by cables to move along the half radius focal sphere. The main advantages of such a design are the absence of delay lines, and the simplicity of the optical train.

\section{Specificities of the OHP prototype and description of its subsystems}

One goal of the $10 \mathrm{~m}$ baseline OHP prototype is to test the whole optical train of Carlina. In the next sections, we describe the optical and mechanical solutions that we found to build a prototype (holding system, metrology gondola, and primary mirror). We discuss the technical choices in order to respond to the required specifications of Table A.1. All the subsystems described in this article are schematically represented in Fig. 2 that gives an overview of the complete experiment (without the focal gondola).

\subsection{Optical design}

\subsubsection{Baselines and position of the mirrors}

The CARLINA design at OHP has a $R=71.2$ m curvature radius ( $R$ is constrained by the maximum space available at OHP for installing the holding system). The focal gondola is on the focal sphere at $\frac{R}{2}=35.6 \mathrm{~m}$. The classical corrector of spherical aberration has a maximum aperture ratio equal to $f / 2$ (Paper I). It means that the maximum baseline at Haute-Provence observatory is $18 \mathrm{~m}$. We decided to start with three mirrors on the ground, forming three baselines of respectively 5, 9, and $10.5 \mathrm{~m}$ (Fig. 2). The $9 \mathrm{~m}$ baseline is oriented N-S and the smaller baseline E-W. These baselines constitute a reasonable intermediate 


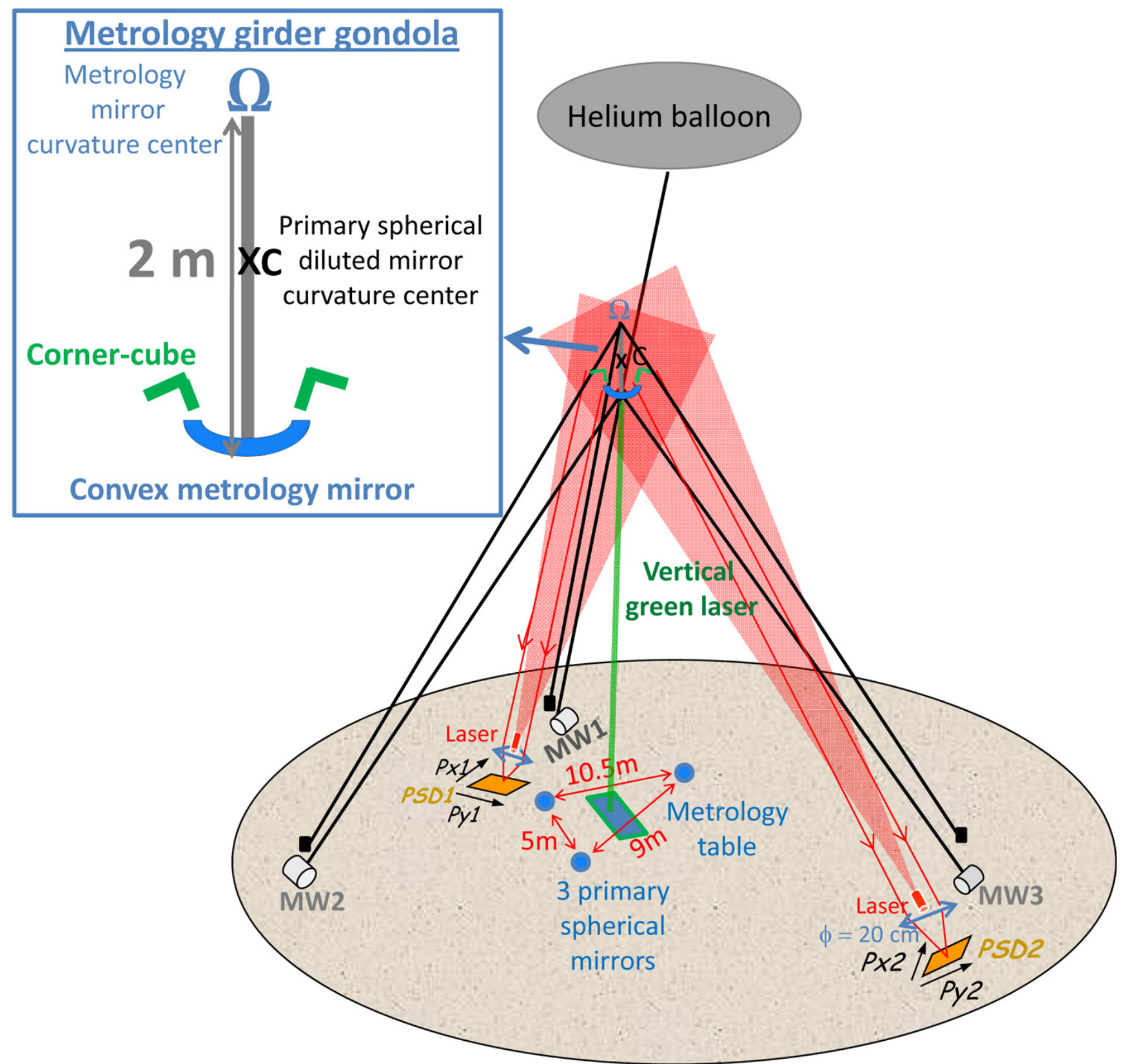

Fig. 2. Schema of the OHP interferometer prototype. In order to show the whole experiment, this is not a scale drawing. The black lines represent the cables. The blue circles symbolize the three primary mirrors at the ground level around the metrology table (Fig. 10). The convex metrology mirror is attached at the bottom of a girder gondola. The helium balloon keeps the cables under tension by pulling to the lower part of this girder gondola. Point $C$ which corresponds to the center of the spherical diluted primary mirror, is at the middle of the girder. $\Omega$ at the top of the girder is the curvature center of the convex metrology mirror. It is passively positioned by three cables attached to the ground. This mechanical design allows the metrology mirror to rotate around its own center of curvature $\Omega$, if the balloon oscillates under the wind. The white cylinders $(M W 1, M W 2$, $M W 3$ ) are the three motorized winches stabilizing the metrology mirror. The devices to measure the displacements of the metrology gondola are also shown: red lasers in front of the $20 \mathrm{~cm}$ lens and corner cubes attached on the metrology gondola (Sect. 5.1.1). For the sake of clarity, the focal gondola under the metrology gondola is not represented.

step towards the mirrors of a future $100 \mathrm{~m}$ aperture scientific project.

\subsubsection{Description of the metrology system}

The primary mirrors have to be aligned (on a sphere of curvature radius $R=71.2 \mathrm{~m}$ for the OHP prototype) with an accuracy about equal to the atmospheric piston, typically one micron. This is the goal of the metrology described in this section.

The principle: the primary segments produce an image of a point source located at their common center of curvature in the metrology gondola (Fig. 1); we adjust the tilt-tip of each mirror by superimposing these images at the curvature center; the piston is adjusted by searching for fringes. The residual piston errors are comparable to the coherence length of the light source and it is adjusted down to the seeing limited value of a few microns, using a source of white light.

In practice, the light source and the camera at the curvature center have been replaced by a convex mirror called a metrology mirror (Fig. 3). The convex metrology mirror creates a virtual image (near point $C$ in Figs. 2, 3) of a light spot produced by a laser located on a metrology table at ground level (Sect. 4.2.4, 


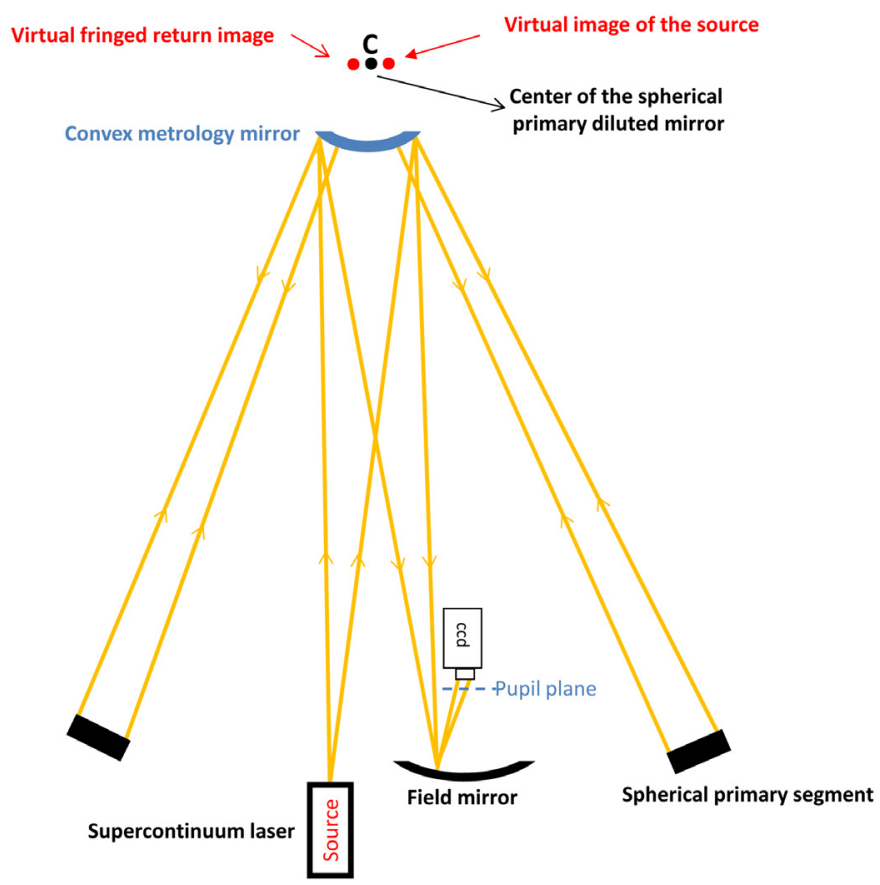

Fig. 3. Schema describing the coherencing technique. A laser source lights up a convex mirror near the curvature center of the spherical diluted primary mirror. The convex metrology mirror creates a virtual image of this source that is seen as a point source by the primary segments. The light comes back on a field mirror where fringes are formed. A CCD is placed a few centimeters after the exit pupil plane, where the diffraction of the small subapertures is high enough to observe the fringes (the beams overlap). This optical design tolerates translation ( $\pm 2 \mathrm{~mm}$ with the OHP prototype optics) of the metrology mirror at the curvature center. With a broadband laser source, finding the white fringe allows us to adjust the piston balance within one micron. On the prototype at OHP, the laser source, the field mirror and the CCD are placed on a metrology table (Fig. 7).

Fig. 7), itself seen as a point source by the primary segments. The primary segments create a virtual fringed return image. The light comes back toward a field mirror on the metrology table where fringes are formed. A CCD is placed a few centimeters after the exit pupil created by the field mirror, where beams are partially superimposed due to diffraction. This optical solution allows lighter equipment to be carried under the helium balloon (only one mirror). It is simpler to use, because there is no need for energy and no need to pilot a camera and lasers from the ground.

\subsubsection{Design of the metrology optics}

The metrology optics to cospherize the primary segments (schematically described in Fig. 3) has been designed with the Zemax software (Fig. 4) in order to accept the oscillations of the convex mirror at the curvature center of the primary $( \pm 2 \mathrm{~mm}$ measured experimentally at the top of a tripod attached to a balloon in a relatively constant wind). We then found that a $600 \mathrm{~mm}$ field mirror is required (for metrology mirror of $1 \mathrm{~m}$ focal length): the fringes cross the $600 \mathrm{~mm}$ field mirror (Fig. 4) with horizontal oscillations of $\pm 2 \mathrm{~mm}$ of the metrology mirror in the wind. We will see that the field mirror has been slightly oversized because we attached the metrology mirror in a such a way that it turns about its own curvature center (Sect. 4.2.3): the horizontal displacements of $\pm 2 \mathrm{~mm}$ are transformed in rotations.
In a conventional optical design, we would place a lens in front of the CCD (Figs. 3, 4) to produce an image of the fringes (field mirror), but in our case there are no optics between the field mirror and the camera. Fringes appear quasistatic on the camera with this optical design because they are in a "pseudo pupil plane" a few centimeters after the exit pupil where light beams are partially superimposed, as diffraction gives rise to significant beam overlap (practically total). For future projects, a tip-tilt mirror could be placed at the exit pupil created by the field mirror, to stabilize the image at the entrance of a "dispersed speckle" piston sensor (Borkowski et al. 2005; Tarmoul et al. 2010) or any other "wavefront sensor" adapted to diluted pupils.

Three sets of fringes always intersect each other at a unique common point (top and bottom images of Fig. 4). Along the line defined by a white fringe, the light beams coming from two apertures are in phase. For mirrors $\mathrm{N}$ and $\mathrm{S}$ (see position of the OHP mirrors in Fig. 10), this translates into the following identity: $\phi_{\mathrm{N}}=\phi_{\mathrm{S}}$ (where $\phi$ is the phase of the light beam from a given mirror). The same principle applies for the white fringe created by the mirrors $\mathrm{N}$ and $\mathrm{W}: \phi_{\mathrm{N}}=\phi_{\mathrm{W}}$. At the intersection between of both white fringes, $\phi_{\mathrm{N}}=\phi_{\mathrm{S}}=\phi_{\mathrm{W}}$. The white fringe created by mirrors S and W (defined by the line where $\phi_{\mathrm{S}}=\phi_{\mathrm{W}}$ ) therefore passes through this intersection. Figure 5 shows the results of the same study as presented in Fig. 4 but with a fourth primary mirror. The fourth mirror is aligned by adjusting its piston screw, until the three sets of fringes linked with this mirror cross the reference triplet (centered on the envelope as described at Fig. 4) in a unique point. This technique is very sensitive because the fringes are very narrow. Without turbulence (for example if we build such an interferometer in space), it should be possible to adjust their position with respect to each other with much better accuracy than the width of one fringe (piston error $\ll 1 \mu \mathrm{m}$ ).

Usually, regular interferometers are densified. The densification shrinks the diffraction envelope. Consequently, the white fringe moves out of the diffraction envelope for a piston error of only a few microns. Fringes are spectrally dispersed (Weigelt et al. 2000; Petrov et al. 2007; Mourard et al. 2009; Tarmoul et al. 2010) both for scientific reasons, and also in order to increase the coherence length in order to be able to detect them even with a large piston error. In our case, we did not need to spectrally disperse the fringes because our metrology works in "Fizeau" mode, and the white fringe can be detected in a large speckle envelope without spectral dispersion, i.e. $\approx 50 \mu \mathrm{m}$ piston error on the primary mirrors at OHP. This is an important and interesting solution of our metrology optical design to coherence the mirrors of an interferometer (see also discussion on the CCFFS in Sect. 6).

\subsubsection{Optical path errors that are not common to focal beam and to metrology beam}

We have described a metrology at the curvature center. In this section, we explain how it is ensured that, if the primary mirrors are coherenced from the curvature center, they are also coherenced for the stellar light. Typically, the accuracy of the metrology at the curvature center (to align the primary mirrors), is equal to the wavelength used for the metrology. The metrology indeed proceeds through the measurement of fringes for which a displacement of one fringe corresponds to an error of one lambda on the wavefront. Also, to be sure that the measured coherencing level at the curvature center is sufficient for observations of stellar objects, it is preferable to use a metrology source with an effective wavelength smaller than or equal to the effective wavelength of the stellar light (middle of the filter bandwidth). 

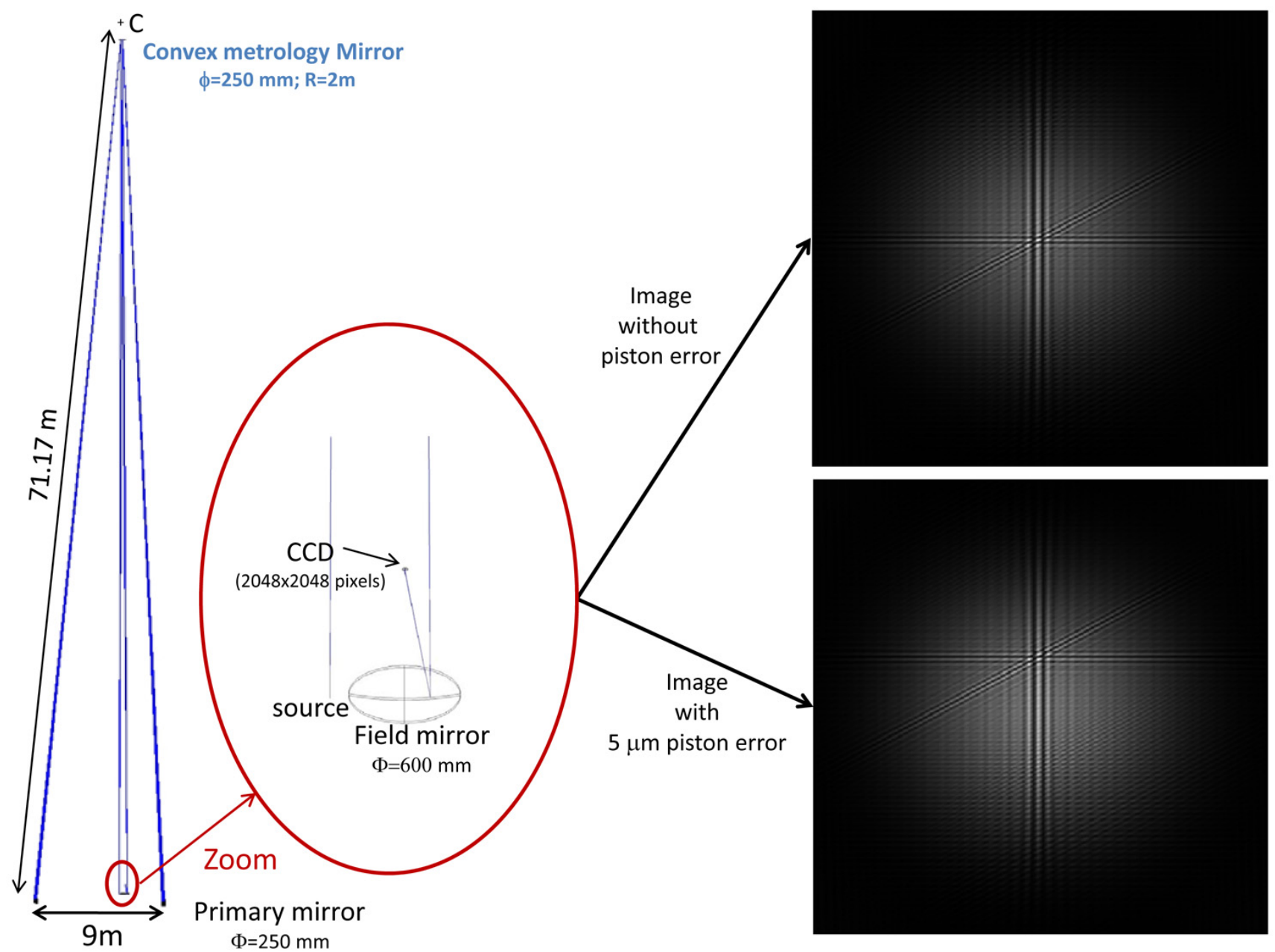

Fig. 4. Optical design of the OHP prototype metrology simulated with the Zemax software. On the left, a scale schematic view similar to that shown in Fig. 3 illustrates the plane including the $9 \mathrm{~m}$ baseline and the $C$ point. In the middle, a zoom of the field mirror is shown: the ray tracing is for a $1 \mathrm{~mm}$ offcenter metrology mirror (horizontal displacement). On the right, the two images give the results of simulations performed, respectively, without and with piston errors (on the $\mathrm{N}$ mirror). In the two cases, the fringes of the three baselines at OHP $(5 \mathrm{~m}, 9 \mathrm{~m}, 10.5 \mathrm{~m})$ are shown.

By using the same wavelengths for the metrology and the stellar light, an error of one lambda on the wavefront (piston error of $\lambda / 2$ for a primary mirror) moves the fringes pattern by one metrology fringe at the curvature center and one stellar fringe at the focal camera. Nevertheless, if we observe in the visible we gain nothing by using a metrology source with an effective wavelength that is much smaller than one micron because the atmospheric turbulence will add more than one micron of piston error to the stellar light.

Also there is no optical surfaces between the primary and the focal gondola that could introduce optical path errors, which would not be seen by the metrology at the curvature center. Only the atmosphere adds a piston error that is not measured by the metrology at the curvature center because the star light does not cross the same atmospheric layers (Fig. 1). In the focal gondola, the corrector of spherical aberration (Mertz 1996) is made of two aspheric mirrors. This device is made of continuous optical surfaces (no independent optics for each beam), and supposes a spherical primary, which is a condition ensured by the metrology at the curvature center. A diamond turning machine has shaped these mirrors with an accuracy much better than one micron, i.e., better than the atmospheric piston error.

To conclude, it is ensured that if the mirrors are "coherenced" (cospherized) from the curvature center, they are also "coherenced" for the stellar light with about the same accuracy (the atmospheric piston $\approx 1 \mu \mathrm{m}$ ). This metrology is equivalent to the active optics of a regular telescope. From this point of view, Carlina looks like a regular telescope working without delay lines. When the primary segments of a telescope are aligned on a parabolic surface or the surface adapted to the conjugation of the telescope mirrors (in our case, a spherical primary + Mertz corrector in the focal gondola) with an accuracy of a few $\lambda$, the stellar light is coherenced at the focal point.

\subsection{Mechanical design}

\subsubsection{The mount of the primaries mirrors}

Each mirror is supported by an hexapod mount made of carbon fiber legs that ensure micrometric stability (Fig. 6). An hexapod has also six freedom degrees that allows adjusting the position of the mirror in rotation and translation. The length of each leg can be adjusted and blocked to position the mirror with one millimeter accuracy. A second micrometric stage allows finely adjusting the position of each mirror (Fig. 6): two micrometric screws control the tip-tilt, and the piston is adjusted using a vertical translation stage under each mirror. Thus, nine parameters (screws) 

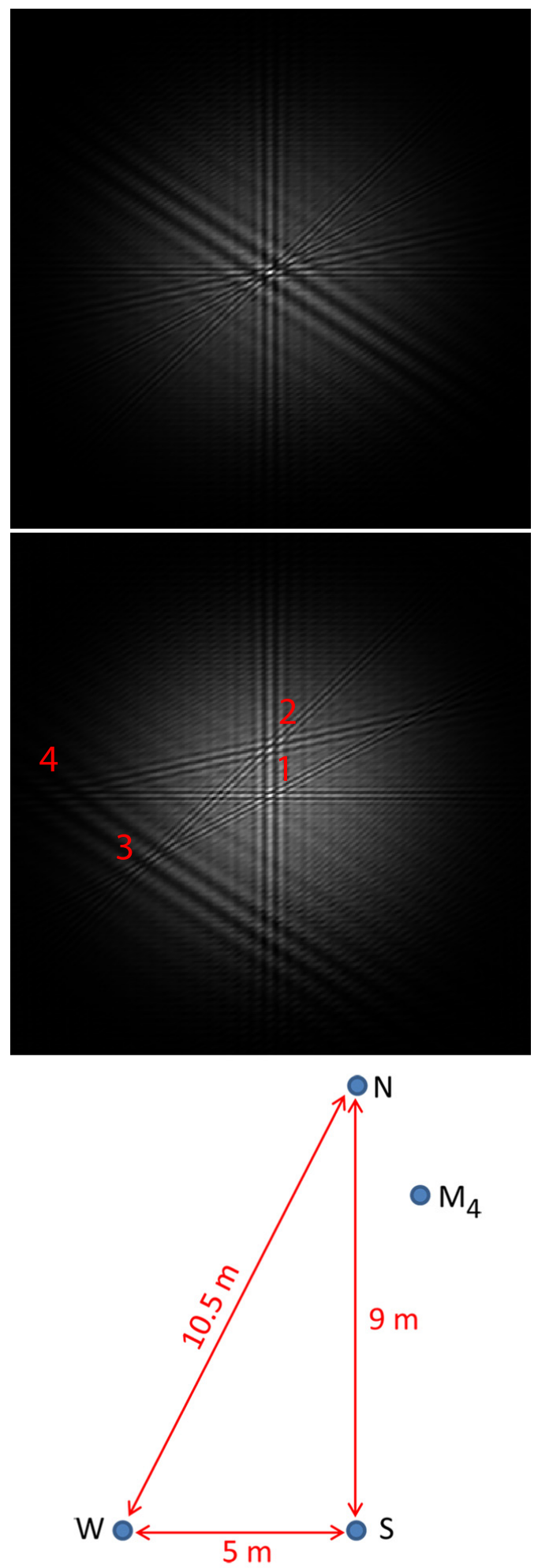

Fig. 5. Same simulation as in Fig. 4 but with a fourth mirror (if $n$ is the mirror numbers: $n(n-1) / 2=6$ fringes and $C_{4}^{3}=4$ triplets). Top: all the mirrors are in phase. Middle: the three mirrors of Fig. 4 are still in phase, but a 3 micron piston error has been added on the fourth mirror $M_{4}$. We wrote numbers in red, which are close the intersection of each triplet of fringes. The number 1 corresponds to the triplet of fringes without piston error of Fig. 4. Bottom: position at scale of the four mirrors.

have to be adjusted within one micron to cospherize the three mirrors around a common curvature center ( $C$ in Figs. 2-4).

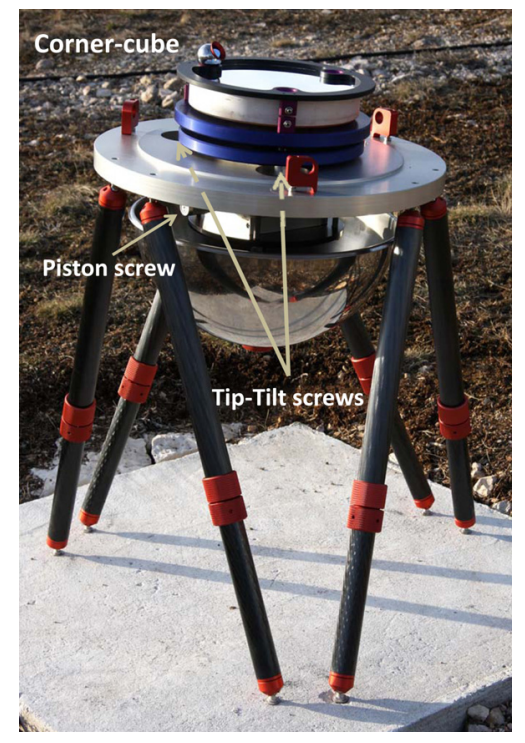

Fig. 6. Picture of the mount that carries one of the primary mirrors. With this hexapod we can position the mirror in rotation and translation. The carbon fiber legs insure a stability better than one micron.

\subsubsection{Holding system}

At OHP, the focal gondola is at $f=35.6 \mathrm{~m}$ and the metrology gondola at $R=71.2 \mathrm{~m}$ above the ground. A 80 to $100 \mathrm{~m}$ high pylon costs about 100 Keuros, that is too expensive for a demonstrator. We then decided to use a captive helium balloon to attach the gondolas above the OHP ground. We are in the most difficult conditions due to the wind resistance of the balloon. The balloon is also a possible solution for a future very large diluted telescope of $500 \mathrm{~m}$ or more. In order to minimize the oscillations of the balloon, it must have a low resistance in the wind with the maximum traction. We used a $12 \mathrm{~m}$ long balloon with eliptic shape (the maximum size for the room where the balloon is stocked). This balloon has a $70 \mathrm{~kg}$ payload.

The gondolas are suspended under the helium balloon with $1.8 \mathrm{~mm}$ diameter cables, made of "PBO" fibers (Orndoff 1995), a member of the polybenzoxazole class of polymers. They have higher tensile strength and Young's modulus than Kevlar: the PBO HM Young's modulus is about 3.8 times that of the Kevlar29 Young's modulus corresponding to $270 \mathrm{GPa}$ for about the same density $\left(1.5 \mathrm{~g} / \mathrm{cm}^{3}\right)$. The total cables weight is about $14 \%$ of the balloon payload.

\subsubsection{The metrology gondola}

Experimentally, we know that the top of the tripod $71.2 \mathrm{~m}$ above the ground can move of several centimeters when wind direction changes, and oscillate horizontally of $\pm 2 \mathrm{~mm}$ in the wind (using $1.8 \mathrm{~mm}$ PBO cables). If the metrology mirror has a horizontal translation movement exceeding $\pm 2 \mathrm{~mm}$ the light goes out of the field mirror (Sect. 4.1.3). We then studied a mechanical way to attach the metrology mirror to limit the oscillation effects (due to the balloon movements in the wind) on the optical beams (Fig. 2). As this one is nearly spherical, if the metrology mirror rotates around its curvature center, the return beams doesn't move on the field mirror on the ground. The design of the gondola allows the translation motions of the metrology mirror (if the balloon oscillates) to be converted into pure rotation around its own curvature center, because it is attached under a girder gondola along its radius of curvature. The length $(2 \mathrm{~m})$ 


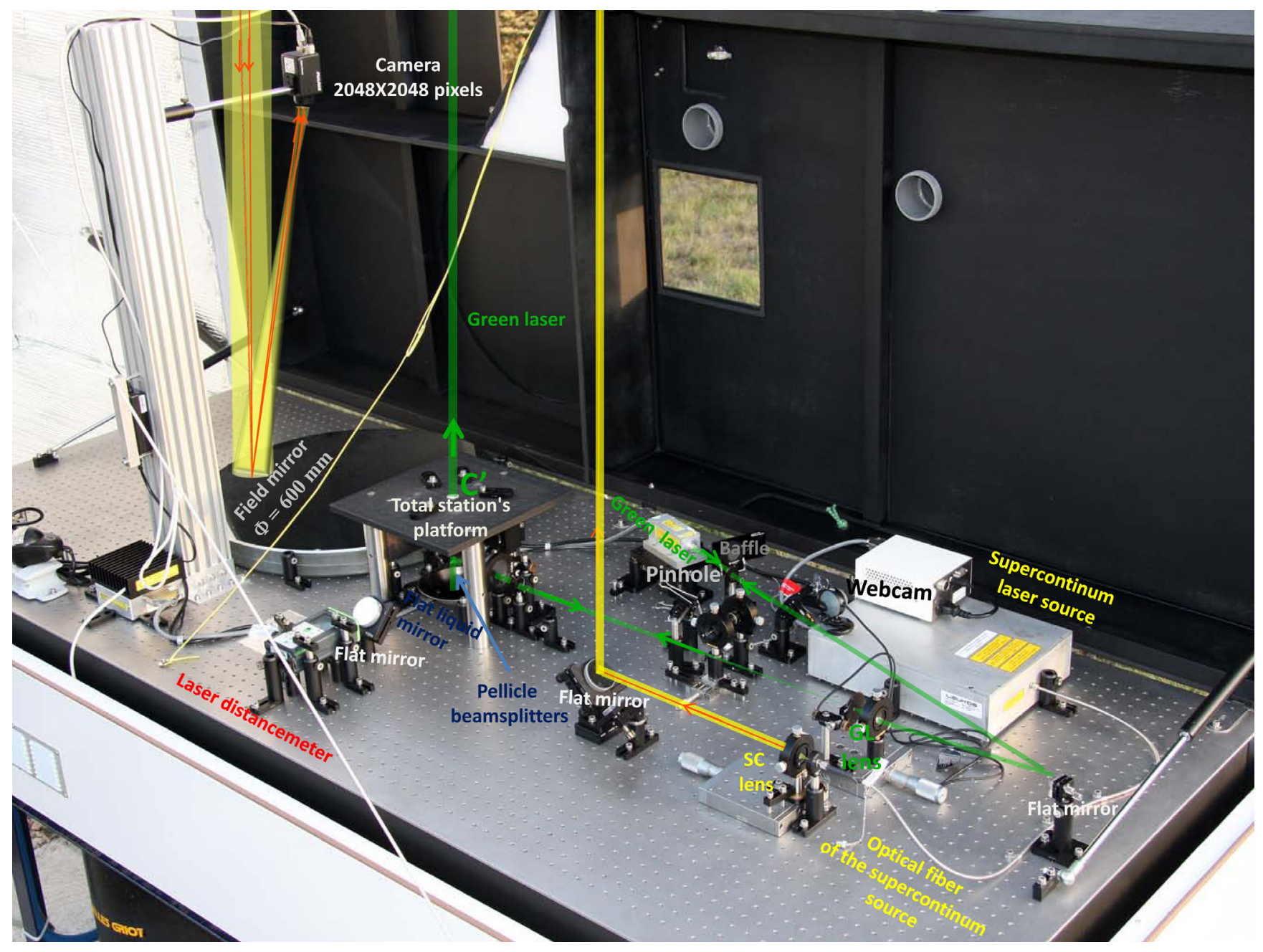

Fig. 7. Picture of the metrology table. A large white hood (on the left in the picture) protects the table from the rain. To protect the optics from humidity and wind, a second black hood is also used (open on this photo to see the optics) when we are making observations. It has several holes to allow laser beams to pass through. A green laser is used as a vertical reference (a principle schema is presented Fig. 9) in order to align the metrology gondola. The laser distance meter measures the altitude of the metrology gondola to a precision of $\approx 1 \mathrm{~mm}$. As described in Figs. 3 and 4, we use the supercontinuum laser to cospherize the primary mirrors; a camera above the $600 \mathrm{~mm}$ field mirror near a pupil plane records the white fringed return image (only visible when the primary segments are aligned).

of this girder is equal to the curvature radius of the metrology mirror (Fig. 8). Two cable tripods are attached at the bottom and at the top of this metrology gondola (Figs. 2 and 8).

The top of the girder is maintained by an unmotorized tripod (yellow upper tripod in Fig. 8). The bottom of the girder carries the metrology mirror and is attached to the balloon pulling on a motorized lower tripod (Figs. 2 and 8). The cables that hold the balloon go through a spacing triangle preventing them from touching the girder gondola. As the varying forces due to balloon oscillations are applied to the girder's bottom, its top end $\Omega$ remains quasistatic, and the metrology mirror rotates around its curvature center $\Omega$, with a reduced effect on light beams. Nevertheless, if the girder pushes to the side, $\Omega$ moves slightly because the forces equilibrium in the top tripod is modified.

In passive mode (when the lower tripod motors are not running), the residual motions of the girder's bottom are $5-30 \mathrm{~cm}$, and less than $1 \mathrm{~cm}$ at the top $\Omega$. Obviously, these residual movements produce negative effects:

- the metrology mirror can move outside the beam if the balloon pulls strongly sideways;
- the metrology fringes fall outside the field mirror (Fig. 4) if the metrology mirror curvature center $\Omega$ is more than $2 \mathrm{~mm}$ offcentered;

- high velocity vibrations can scramble the metrology fringe detection;

- the tripod's motion induces oscillations of the focal gondola that perturb the guiding and scramble the observed stellar fringes.

To solve these problems, the metrology mirror (girder's bottom) is actively stabilized (Sect. 5.1.1).

\subsubsection{Metrology table}

The experiment (girder metrology gondola and primary mirrors) has been aligned from a stable metrology table (Figs. 7, 10). This table is equipped with:

- a standard high precision industrial measurement device (ZEISS total station) to position the mirrors. The so called total station consists in a theodolite combined with a laser distance meter; 


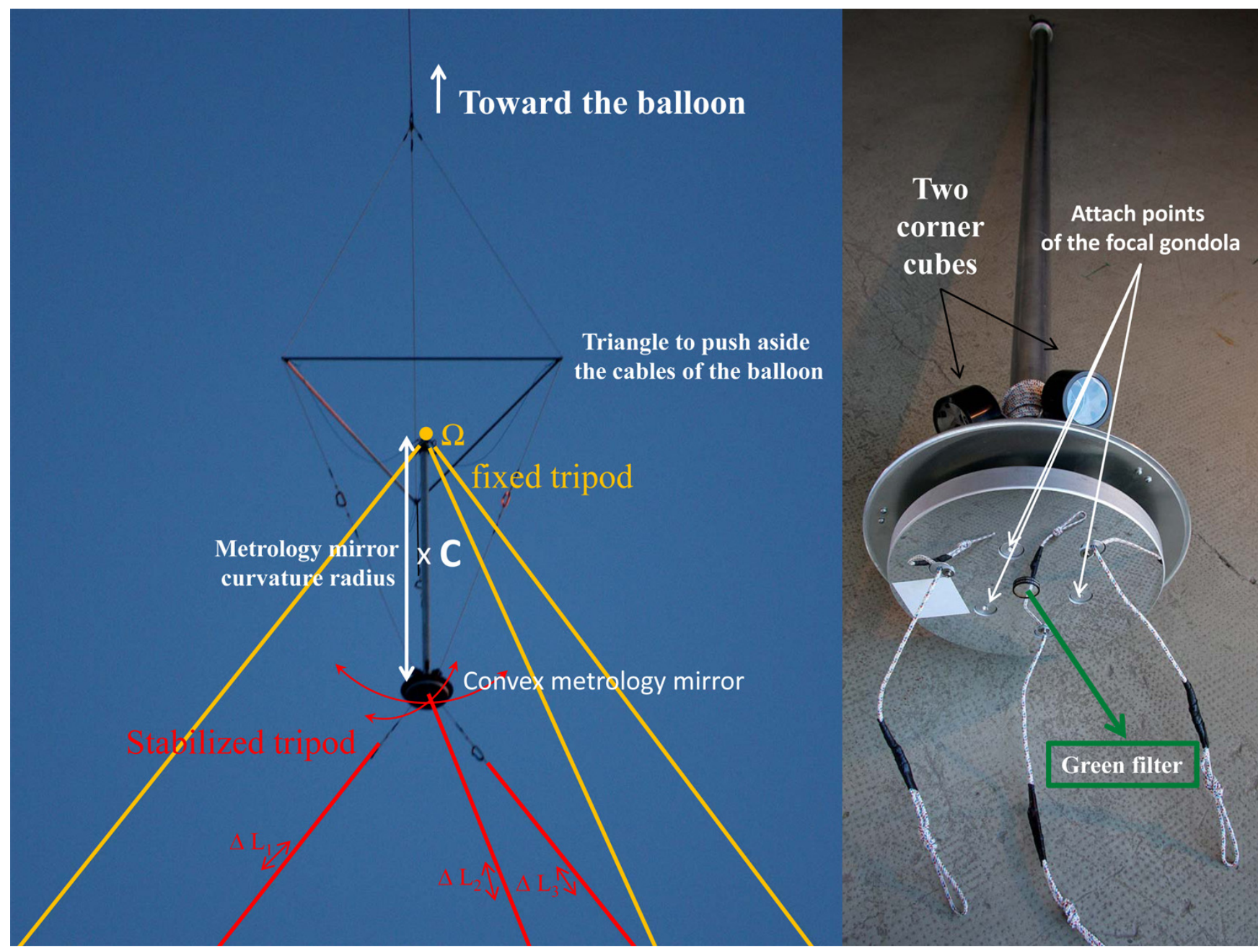

Fig. 8. On the left is shown a photograph of the girder metrology gondola at the curvature center of the diluted primary mirror. We drew in yellow and red the cables with low contrast on the picture. The convex metrology mirror is attached in such a way that it mainly turns around his own curvature center $\Omega$ when the balloon oscillates in the wind. The bottom tripod (in red) is stabilized. On the right, a close view of the girder gondola: two corner cubes attached at the bottom of the girder are used by the servo loop system (Sect. 5.1.1). The green filter at the center of the mirror creates a shadow on the metrology table on the ground. It improves the contrast of the white fringe return image. Behind the green filter, a circular sight is used to align vertically the gondola. Six holes have been drilled in the metrology mirror using a diamond tool. The bottom tripod cables (red) converge through three holes toward the point where the balloon is attached. The three attachment points of the focal gondola are oriented toward the middle of the girder $(C$ point $)$ in such a way that it will turn around the curvature center of the diluted primary mirror. The laser distance meter's light (Fig. 7) is reflected on the white sheet on the left of the metrology mirror.

- a vertical green laser used as a reference to align the metrology gondola;

- a LEUKOS-SM (class 3b) supercontinuum laser source (for alignment of the primary mirrors with one micron accuracy) delivering $96 \mathrm{~mW}$ (pulse width $<1 \mathrm{~ns}$ ) with a wide spectrum from $300 \mathrm{~nm}$ to $2400 \mathrm{~nm}$. IR has been removed with a wide band hot mirror (reflecting $\approx 100 \%$ of the light beyond $750 \mathrm{~nm}$ ) placed at the fiber output;

- a laser distance meter to measure the altitude of the metrology gondola.

In the next sections, we describe how we have aligned all the optics from the metrology table.

\section{Alignment}

All the optics and mechanical parts have been positioned from the point $\left(C^{\prime}\right)$ defined as the vertical projection of the curvature center of the primary sphere (Point $C$ in Figs. 2-4) onto the metrology table on the ground (Fig. 7).

\subsection{Alignment of the metrology gondola}

The metrology gondola is positioned vertically above $C^{\prime}$ thanks to the vertical green laser of the metrology table (Figs. 7, 9). The green laser is vertically adjusted within one to two arcsec thanks to a silicon flat liquid mirror on the metrology table (Fig. 7). A few centimeters above this liquid mirror, a tilted pellicle beam splitter (inclination at $45^{\circ}$ ) reflects the light of the $150 \mathrm{~mW}$ green laser $(\lambda=532 \mathrm{~nm})$ exactly toward its own source in such a way that the other part of the beam reflected on the liquid mirror goes vertically to the metrology gondola (Fig. 2).

Initially, we observe the metrology mirror $(\approx 71 \mathrm{~m}$ above the metrology table) with a small telescope located on the side of the metrology table, and we adjust the length of the lower tripod cables (red cables in Fig. 8) until the middle of the mirror approximately intersects the green laser beam (Figs. 2 and 8). The green laser is focused from the metrology table (using the GL Lens of Fig. 7) to the center of the metrology mirror $(\Omega$ in Fig. 8) so that the return spot appears as small as possible on the ground. Then, we adjust the cable length of the top tripod 


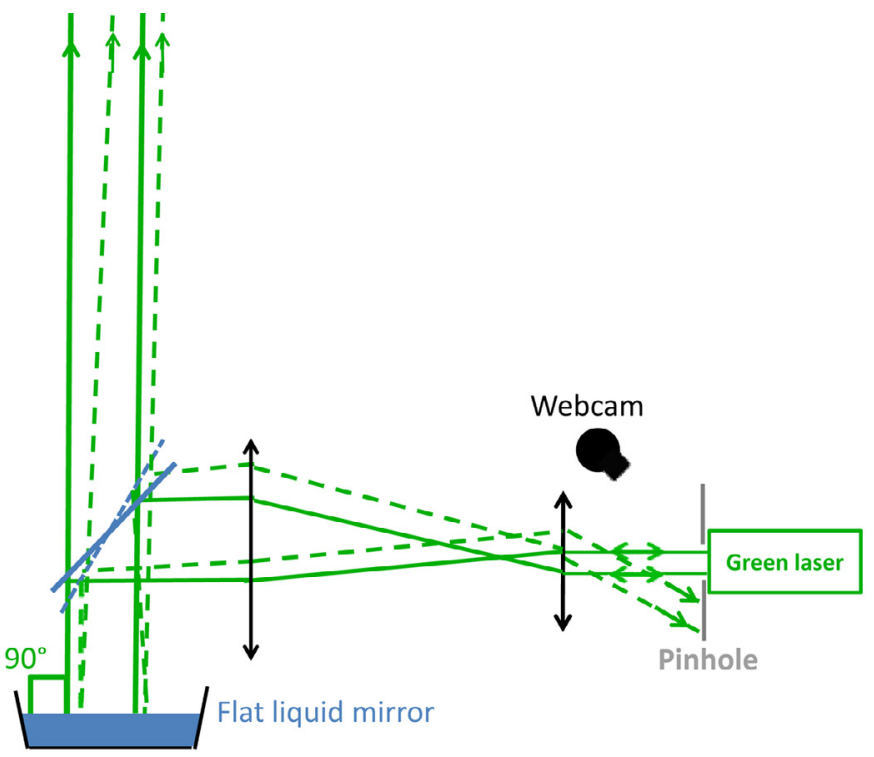

Fig. 9. Principle for creating a vertical laser reference: a pellicle beam splitter above a flat liquid mirror is tilted in a such a way that the green laser goes back through a pinhole filmed by a webcam i.e. the fraction of the green laser light transmitted is perfectly vertical and can be used as a reference to align the metrology gondola above the $C^{\prime}$ point (Fig. 7) of the metrology table. The dashed lines show the light rays if the beam splitter is misaligned. The reached accuracy is within one to two arcsec.

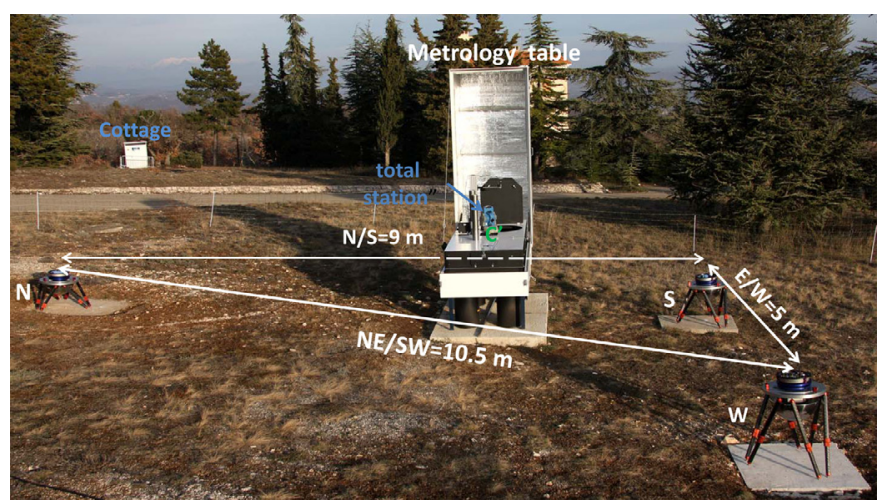

Fig. 10. Photo of the Carlina mirrors. We see the three baselines of $9 \mathrm{~m}$, $5 \mathrm{~m}$, and $10.5 \mathrm{~m}$ oriented respectively N/S, E/W and NE/SW, around the metrology table described in Fig. 7. The black hood is closed, and the trap door of the field mirror is open as during the observations. The total station helps to position the primary mirrors before observation. On this picture, the total station points to a corner cube positioned on the edge of the W mirror (Fig. 6).

(yellow cables in Fig. 8) to send back the green laser light toward $C^{\prime}$ to ensure that the metrology mirror curvature center $\Omega$ is accurately positioned vertically above $C^{\prime}$. A sight is drawn on the center of the metrology mirror disk (Fig. 8), and a diffractive image of this sight is thus visible in the return spot. An accurate alignment is obtained by manipulating the lower tripod's motorized winches, to center this image in the return spot (the sight is vertically positioned above $C^{\prime}$ ), ensuring that the girder gondola is perfectly vertical. A laser distance meter measures the altitude of the metrology mirror with one millimeter accuracy (Figs. 7 and 8 ). The vertical tolerance is $\Delta_{z}=5 \mathrm{~mm}$ for $10 \mathrm{~m}$ baselines and the atmospheric turbulence at OHP (Table A.1). We iterate several times this process of alignment until the metrology gondola is vertically oriented above the metrology table and at the correct altitude.
Once the metrology gondola is correctly positioned, we turn on a servo loop.

\subsubsection{Servo loop system to maintain the metrology gondola alignment}

With a wireless anemometer attached to the balloon, we measured (as seen from the ground with a small telescope) that an horizontal wind speed up to $15 \mathrm{~km} / \mathrm{h}$ shifts horizontally the metrology mirror of about $30 \mathrm{~cm}$. These large movements are slow, due to balloon inertia ( $30 \mathrm{~s}$ to a few minutes). Wind buffeting at typically $1 \mathrm{~Hz}$ induces displacement of a few millimeters. We have developed a Servo loop to correct for these motion. Note that above $\approx 20 \mathrm{~km} / \mathrm{h}$ wind speed, the experiment goes down (a cable of the tripod is slackened) and it is not possible to work even with a servo loop. This result could probably be improved a lot using a balloon with better aerodynamical behaviors.

The stability of the metrology gondola is achieved by means of the lower tripod of the girder (red cables in Fig. 8). The lengths of the tripod cables are accurately controled by three computer driven winches ( $M W 1, M W 2, M W 3$ in Fig. 2). At ground level, two red lasers light up two cornercubes (Fig. 8) attached on the girder gondola (close to the metrology mirror). The cornercubes return the light toward the sources (Fig. 2), and the $20 \mathrm{~cm}$ large lens, behind each laser creates an image of the cornercubes on a position sensitive detector (PSD). We measured a $50 \mathrm{~ms}$ time response to longitudinal constraints for a $100 \mathrm{~m}$ Kevlar cable (Appendix B). We therefore operate the servo loop at $10 \mathrm{~Hz}$ : each PSD gives two coordinates $\left(P_{x}, P_{y}\right)$ of the image photocenter sampled at $300 \mathrm{~Hz}$ and down sampled at $10 \mathrm{~Hz}$ after passing through an antialiasing filter. An algorithm determines the girder position (position of the corner cubes). Three computer-controlled winches bring back the girder toward its initial position ( $M W 1, M W 2, M W 3$ in Fig. 2).

We use an algorithm derived from adaptive optics devices (Jacubowiez et al. 1997). It is a method based on a singular value decomposition (SVD) of the rectangular matrices. Such a method works even if the positions of the motor winches and of the girder gondola are not a priori fully determined.

The lower tripod summit motions (metrology mirror in Fig. 8) are linked with the displacements of the photocenters (image of the corner cube) on both PSDs (Fig. 2) by the linear equation system:

$\Delta \boldsymbol{L}=M \cdot \Delta \boldsymbol{P}=\left(\begin{array}{l}\Delta L_{1} \\ \Delta L_{2} \\ \Delta L_{3}\end{array}\right)=\left(\begin{array}{llll}m_{11} & m_{12} & m_{13} & m_{14} \\ m_{21} & m_{22} & m_{23} & m_{24} \\ m_{31} & m_{32} & m_{33} & m_{34}\end{array}\right)\left(\begin{array}{l}\Delta P_{x 1} \\ \Delta P_{y 1} \\ \Delta P_{x 2} \\ \Delta P_{y 2}\end{array}\right)$.

Determination of the interaction matrix $M$ is described in Appendix D. It is used in a velocity loop proportional integral derivative (PID) to drive the three motor winches at $10 \mathrm{~Hz}$ :

$\boldsymbol{V}(n)=K_{\mathrm{p}} \cdot \Delta \boldsymbol{L}(n)+K_{\mathrm{I}} \sum_{i=1}^{n} \Delta \boldsymbol{L}(i)+K_{\mathrm{d}}[\Delta \boldsymbol{L}(n)-\Delta \boldsymbol{L}(n-1)]$

where $n$ is the discrete step at time $t, \boldsymbol{V}$ the command velocity sent to the motor winches, $K_{\mathrm{p}}$ the proportional gain, $K_{\mathrm{I}}$ the integral gain, and $K_{\mathrm{d}}$ the derivative gain.

In active mode, bottom (metrology mirror in Fig. 8) and top ( $\Omega$ in Fig. 8) movements of the girder are reduced respectively within $\approx 5 \mathrm{~mm}$ and $\approx 0.3 \mathrm{~mm}$ (see Sect. 6 ). The residual servo 
loop errors are mainly due to the gondola inertia, the cable response (delay for the gondola to move when we pull on a cable), the atmospheric turbulence (which limits the accuracy with which the position of the corner cubes can be measured), the electronic noises, etc.

To optimize the system, it would be interesting to measure the closed loop bandwidth of the system (rejection function). To achieve that, it would be necessary to implement a recording of the PSD signals and of the correction sent to the motors in the servo loop software. Those data should be considered in relation with recorded wind speed measurements. Moreover, several anemometers at different levels would probably be necessary, as the wind speed distribution changes significantly in different wind systems: wind gust, wind from N, S, partly cloudy, etc. We have already observed a wind of $10 \mathrm{~km} / \mathrm{h}$ at the level of the balloon and practically zero at the ground. Moreover, the oscillation frequencies will probably change with the focal gondola payload (not installed yet). As described at the top of this section, the servo loop works at $10 \mathrm{~Hz}$ (see Appendix B). It could be interesting to optimize it. In the future, such a wind dependent full optimization could be justified for a large scale diluted telescope project. However, such a detailed characterization goes beyond the scope of this paper. Here, we demonstrate that the servo loop system is largely good enough to get stable metrology fringes under $20 \mathrm{~km} / \mathrm{h}$ wind conditions.

\subsubsection{Alignment of the primary mirrors}

The total station is placed in the middle of the metrology table (point $C^{\prime}$ in Fig. 7). It is used to point a corner cube (centered on a sphere within $\approx 10 \mu \mathrm{m}$ ) that is placed successively at three points on the edges of each mirror (Figs. 6, 10). Two manual micrometer screws allow adjustment of the tip-tilt. The piston is adjusted by a micrometric vertical translation stage.

\subsubsection{Alignment of the primary mirrors with one micron accuracy}

We used the LEUKOS-SM (class 3b) supercontinuum laser source (Fig. 7). We adjusted the tip-tilt of each primary mirror in order to superpose the three spots (images of the supercontinuum source created by each primary mirror) on the field mirror (Figs. 3, 4, and 7). Then, we searched for the fringes in $25 \mu \mathrm{m}$ steps, first using the vertical translation stage of the $\mathrm{W}$ mirror (piston screw in Fig. 6). Finally, the piston of the $\mathrm{N}$ mirror was adjusted to find the N-S and the N-W fringes. Fringes have been detected by scanning less than $200 \mu \mathrm{m}$. It shows that the total station is enough accurate to ensure a good prealignment. The total stations and distance meter technologies are in constant progress. In the future, by replacing the supercontinuum source by a two-mode laser telemetry (Courde et al. 2009; Courde et al. 2010), it could be possible to position the primary mirrors within one micron without searching for the white fringes (present technology).

The piston of the $\mathrm{W}$ and $\mathrm{N}$ mirrors are finely adjusted to center the intersection of the three set of fringes close to the middle of the speckle image (Fig. 11). It is remarkable that Fig. 11 looks like simulated fringes (Fig. 4) but with additional turbulence (speckles). The same procedure can be used to adjust the tiptilt and piston of any additional mirror, by centering the fringes in the speckle image, and at the crossing point of all the fringes (Fig. 5).

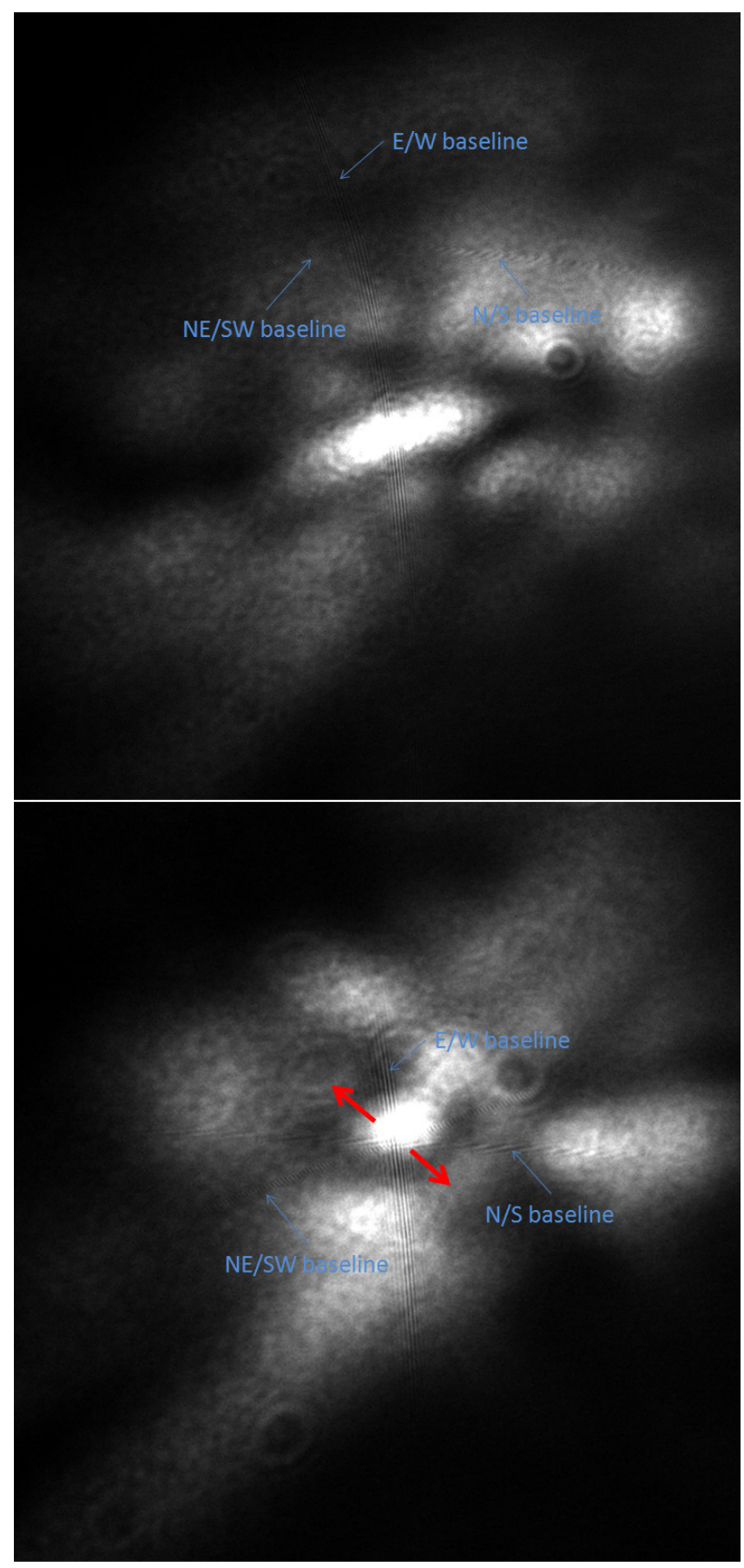

Fig. 11. Fringes from the three baselines. Top, $50 \mu \mathrm{m}$ piston error on the $\mathrm{N}$ primary mirror. Bottom, fringes are centered: the three mirrors are cospheric within a few microns (not possible to have a better adjustment due to atmospheric turbulence). Fringes are subsampled on the pixels of the CCD for larger baselines (N/S, NE/SW) and they appear with a moire pattern. To avoid the moire, fringes are sufficiently stable to be magnified by a factor 10 (using a lens in front of the CCD) but the speckle image would become larger than the CCD. We have checked that the moire pattern does not disturb the accuracy needed to center the fringes (Fig. 12). The red arrows indicate the dominant direction of the oscillations of the fringes on the CCD.

\section{Results}

The optomechanical systems described in this paper has been tested following four main steps:

- Alignment of the metrology gondola (cables length adjustment, test of the vertical green laser, etc.). 
H. Le Coroller et al.: Tests with a Carlina-type diluted telescope
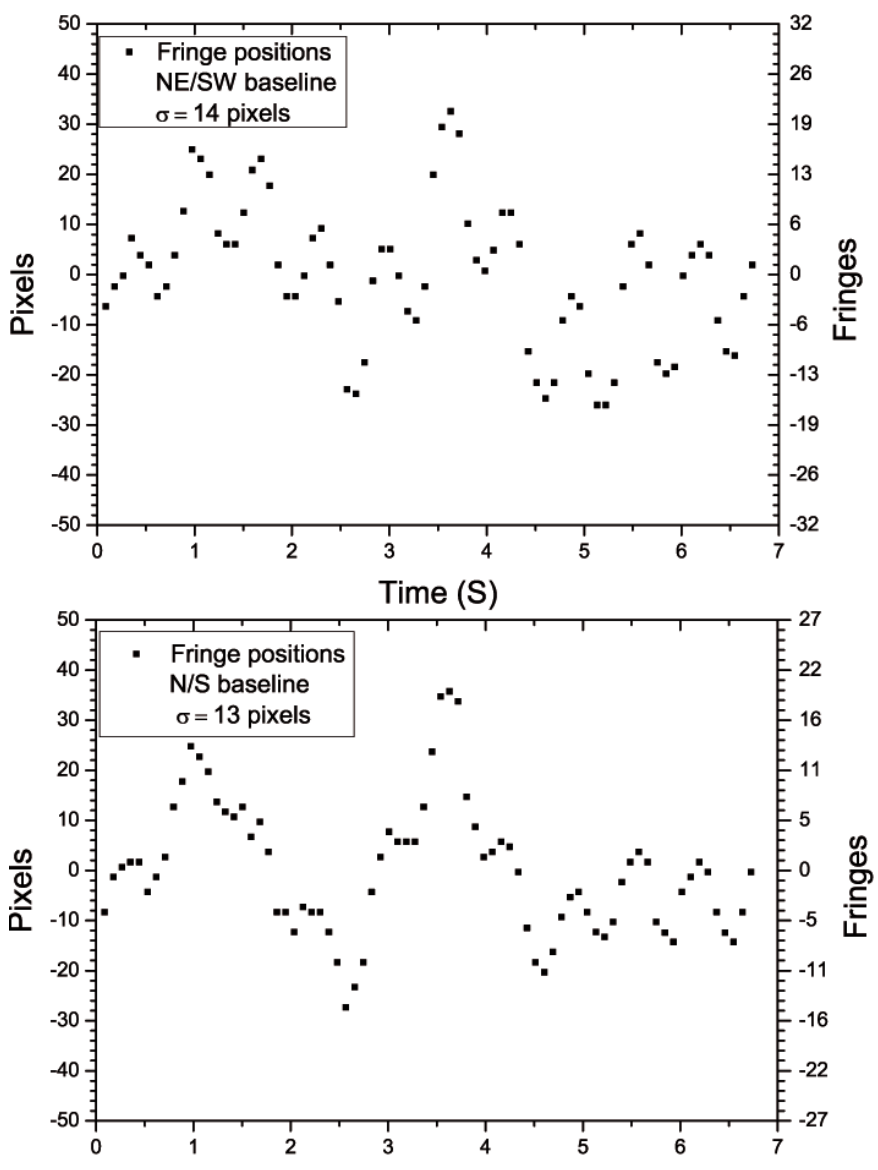

Time (S)

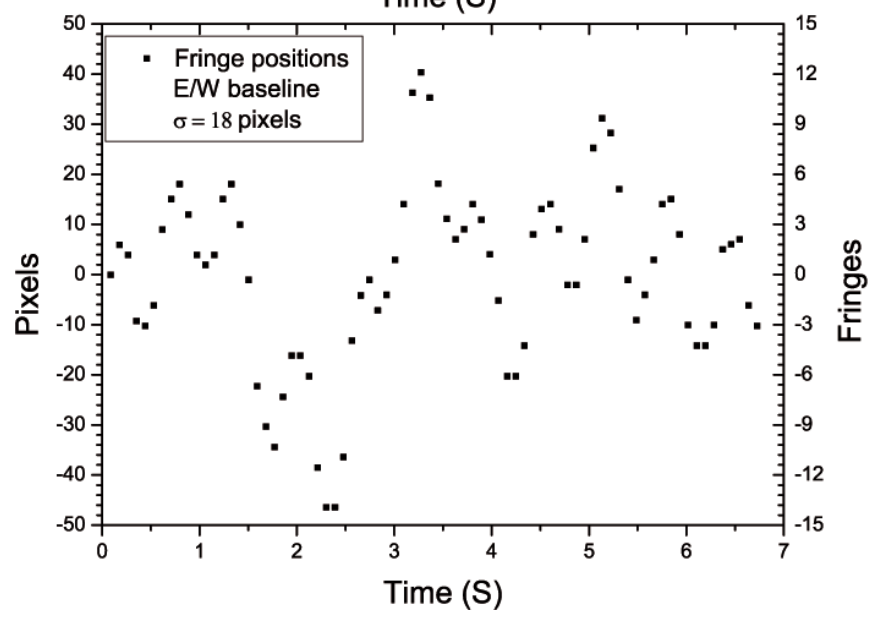

Fig. 12. Displacement of the fringes (in the direction perpendicular to the fringes) from the three NE/SW, N/S and E/W baselines (Fig. 10). On the left, scales are in pixel; on the right, in fringes. One fringe equals $1.56,1.82$, and 3.28 pixels, respectively, for the NE/SW, N/S, and $\mathrm{E} / \mathrm{W}$ baselines.

- Servo loop (interaction matrix creation, PID parameters, bug corrections, etc.).

- Primary mirrors alignment and fringes detection.

- Characterization of the system stability.

A $\log$ of the completed stages is provided in Appendix C. Beside the 380 fringe images $(5 \times 76$ frames recorded on February 23, 2011) to characterize the system stability (as shown in Figs. 12 and 13), we also recorded other data. However, these additional fringe frames don't constitute a coherent data set, and we
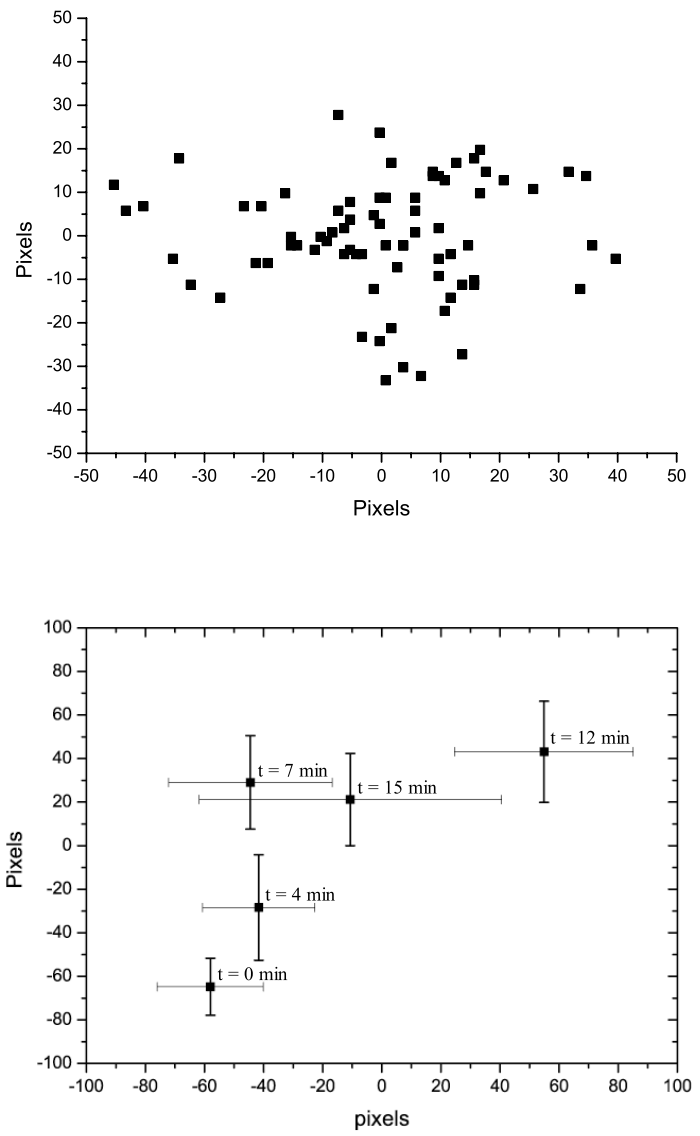

Fig. 13. Top: position of the fringes center (intersection of the three fringes systems) on the CCD during $\approx 7 \mathrm{~s}$ (76 exposures of $1 \mathrm{~ms}$ ). Bottom: each point is the mean position of the fringes center over $7 \mathrm{~s}$ (ex: the point at $t=0 \mathrm{~min}$ is the mean of the top graph). The data have been recorded the 23/02/2011 during 15 min (see log Appendix C).

therefore don't show these results here. Among the numerous measurements performed with the prototype, we mention fringe imaging with only one baseline, piston adjustment of the $\mathrm{N}$ mirror (see e.g. Fig. 11), fringe measurements after interruption and restart of the servo loop, etc. In total, $21 \times 76=1596$ images were recorded without losing fringes during tests lasting for four hours.

\subsection{System stability}

In order to study the system's stability, we have plotted the fringe positions on the metrology camera, as a function of time (Fig. 12). First, we tried to observe the fringe motions relative to the diffraction envelopes images of the subapertures. However, we realized that the photocenter of the diffraction envelopes moves on the camera much more than the fringes. This means that the diffraction envelope photocenter displacement is dominated by the atmosphere (tip-tilts and speckle effects) and not by the girder gondola oscillations. Indeed, the fringes and the diffraction envelopes all move together with the gondola oscillations, while the atmospheric tip-tilt on a subaperture moves only the photocenter diffraction envelope (not the fringes) on the CCD. 
We then measured the fringe displacements relative to the camera pixels. Figure 12 shows that the fringes oscillate around a fixed position during $\approx 7 \mathrm{~s}$, the time needed to fill the PC memory ram (76 exposures at 11.3 images per second over $2048 \times 2048$ pixels). Fringe residual motions are due to the metrology gondola residual oscillations and the atmospheric piston. In Fig. 12, we see that the maximum oscillations are about equal to \pm 220 microns ( \pm 30 pixels). The fringes oscillate in a dominant direction (Fig. 11), probably linked with the wind direction, showing that their motions are correlated well with a metrology gondola oscillation. If their motions were dominated by the turbulence (differential piston on the primary mirrors), their displacement on the CCD would be in random directions. This rectilinear oscillation explains that the three curves (Fig. 12) look very similar in shape and amplitude. These three curves can be interpreted as the projection of a nonuniform rectilinear motion of a point (the intersection of the three fringes) on three axes: the axes perpendicular to the fringes, i.e in the direction of the baselines. By definition, a rectilinear motion of a point projected on any axis gives the same curve in shape on each axis. The three curves of Fig. 12 have about the same amplitude because the fringes oscillate in a direction very different from all the projection axes (see Fig. 11).

The results of the study of the system stability over a longer duration (15 $\mathrm{min}$ ) are presented in Fig. 13. The position of the fringes are averaged over seven seconds at each point in the lower panel of Fig. 13 and then the motion of the fringe center is only due to the mechanics (turbulence averaged in seven seconds). The fringes have moved by $\approx 120$ pixels $(\approx 0.9 \mathrm{~mm})$ during 15 min (Fig. 13). With the Zemax software, we evaluated that a displacement of the fringes of 120 pixels on the metrology CCD corresponds to a motion of $\Delta_{\Omega(x, y)}=250 \mu \mathrm{m}$ of the top of the metrology gondola; i.e., we stabilized the center of curvature of the metrology mirror within $250 \mu \mathrm{m}$ during $15 \mathrm{~min}$ ! This is very satisfactory considering that some of optical components of the system are suspended under a balloon. The optics (field mirror, CCD size, etc.) were dimensioned to tolerate 16 times larger displacements $(\Delta= \pm 2 \mathrm{~mm}$ as given in the specifications of Table A.1). In fact, we never lost the fringes for four hours starting from the moment that we found them on the CCD.

Moreover, the fringes remains centered on the envelope (subapertures image) for $15 \mathrm{~min}$ at least, without touching the primary mirrors. This demonstrates the excellent stability of the mechanical supports, as well as the stability of the ground itself i.e. fringes are centered in the envelope with a few fringes accuracy. This result is well in the specifications of Table A.1.

For a scientific diluted telescope, the tens (or hundreds) of mirrors will be motorized and it will be easy to track the possible slow drifts. We will have to develop an algorithm adapted to track the mean position of the fringes. A cross correlation of the fringes could be a good solution to explore this algorithm. We could call this new fringe sensor for diluted pupils: the CCFFS, for cross correlation Fizeau fringe sensor.

To conclude, we have centered the fringes on a mean position in the envelope of diffraction of the subapertures (accuracy of a few fringes). Note that our metrology is equivalent to an active system and cannot be used as adaptive optics because the metrology laser beams do not follow the same optical path as the star light (see Sect. 4.1.4). Adaptive optics will have to be implemented in the focal gondola (could use a Fizeau fringe sensor, such as the CCFFS proposed in this paper). Then, it will be possible to make longtime exposures in order to increase the sensitivity.
We have demonstrated that it is possible to align the primary mirrors with a metrology system attached at the center of curvature under a captive helium balloon. This is an important step toward demonstrating the possibility of building such an interferometer using a spherical diluted primary mirror. In Appendix E, we discuss how to search for sites where such a project could be built and how to define the array of primary mirrors (number of mirrors, pupil redundant or not, etc.) depending the science goals. Finally, we propose to build a scientific demonstrator that we will call the LDT. Theoretically, an LDT working with 100 subapertures of $25 \mathrm{~cm}$ diameter should be able to observe objects as faint as $m_{v}=15$ (Le Coroller et al. 2010; De Becker et al. 2011). The limiting magnitude as a function of the mirror surface will be determined experimentally when our prototype is operating with its focal gondola.

\section{Conclusions}

The main result of this work is the demonstration of the capabilities and performances of an original metrology system designed for aligning the primary elements of a diluted spherical mirror under a helium balloon. This is an important step that demonstrates the feasibility of an interferometer with a spherical diluted mirror. Active optics is also an important step toward implementing adaptive optics.

We have developed the servo loop and the metrology to cospherize three mirrors with a spacing of approximately $10 \mathrm{~m}$ on a $71.2 \mathrm{~m}$ curvature radius sphere. The servo loop stabilizes a metrology mirror (near the curvature center of the diluted primary mirror $\approx 71 \mathrm{~m}$ above the ground) with an accuracy better than $5 \mathrm{~mm}$, and within 250 microns for its curvature center. The optomechanical design also allows putting heavy metrology devices (lasers, camera, optics, etc.) on a table at ground level. The metrology fringes are observed behind a field mirror in a pseudopupil plane where they are stable. We have fully responded to the specifications of Table A.1. In particular:

- Stability of the mounts that carry the primary mirrors $\approx 1 \mu \mathrm{m}$. - Stability of the curvature center of the metrology mirror under the helium balloon, demonstrated in this paper over $15 \mathrm{~min}: \Delta_{\Omega(x, y)}=250 \mu \mathrm{m}$ and $\Delta_{z}<5 \mathrm{~mm}$.

To our knowledge, this is the first time that a supercontinuum laser source is used in order to equalize the optical paths of an astronomical interferometer. Such an intense source provides fringes with a high $S / N$ ratio that allows the primary segments to be positioned very accurately, down to the seeing limited value. This was made possible by prepositioning the segments using a total station. We note that future laser telemetry technologies (Courde et al. 2009) could be alternative solutions to position the mirrors of diluted telescopes or interferometer delay lines.

In a future article, we will report on observations made with a focal gondola currently under construction. We will test the optical train from end to end (Mertz Corrector, pupil densifier, etc.) and will clarify experimentally the limiting magnitude. We will measure the stability of this gondola under the stabilized tripod (described in this article). Carlina with the MMT (Carleton 1979) and LBT (Kim et al. 2010) could constitute a new family of telescopes called diluted telescopes. We propose to build in the next 10 years a scientific demonstrator with an aperture of $\approx 100 \mathrm{~m}$ that we will call the LDT. In a second stage, a Very LDT could be installed in an area with extremely good seeing conditions.

Because there are no simple solution, the studies on the compact arrays of mirrors positioned on a sphere (as proposed here) 
Table A.1. Carlina specifications.

\begin{tabular}{|c|c|c|c|c|}
\hline \multirow[t]{2}{*}{ Parts } & \multirow[t]{2}{*}{ Characteristics } & \multicolumn{3}{|c|}{ Specifications } \\
\hline & & High level & Low level & OHP prototype \\
\hline $\begin{array}{l}\text { Primary } \\
\text { mirror }\end{array}$ & $\begin{array}{l}\text { Numerous spherical mirrors, } \\
\text { all with the same curvature } \\
\text { radius. }\end{array}$ & $\begin{array}{l}\text { - Mirrors aligned on a sphere } \\
\text { (coherencing). } \\
- \text { The numbers of mirrors } \\
\text { and their positions have to } \\
\text { be adapted to the science tar- } \\
\text { gets. }\end{array}$ & $\begin{array}{l}\text { Piston and Tip-Tilt of each } \\
\text { mirror must be controlled } \\
\text { with an accuracy of one } \lambda \text {. }\end{array}$ & $\begin{array}{l}-3 \text { mirrors; } R=71.2 \mathrm{~m} \\
- \text { Bases: } 5 \mathrm{~m}, 9 \mathrm{~m}, 10.5 \mathrm{~m} \\
\text { - Mounts stability and micro- } \\
\text { metric screws accuracy } \\
\approx 1 \mu \mathrm{m}\end{array}$ \\
\hline $\begin{array}{l}\text { Focal } \\
\text { gondola }\end{array}$ & $\begin{array}{l}\text { Used to obtain stellar images: } \\
\text { Constituted with a spherical } \\
\text { aberration corrector (Mertz), } \\
\text { a guiding system, and a focal } \\
\text { instrument (densifier, AO, } \\
\text { etc.). }\end{array}$ & $\begin{array}{l}\text { - Track the star on the focal } \\
\text { sphere }(R / 2) \text {. } \\
\text { - The drift during the track- } \\
\text { ing has to be smaller than one } \\
\text { fringe per exposure time. } \\
\text { - Have to be positioned at the } \\
\text { correct focus. }\end{array}$ & $\begin{array}{l}\text { - Maximum tracking veloc- } \\
\text { ity } 7.3 \times 10^{-5} \frac{R}{2} \\
\text { - Velocity drifts }<\lambda f / B \text { per } \\
\text { exposure time } \\
- \text { Focus tolerance (spots } \\
\text { shifted by half their size) } \\
\Delta F \approx \pm\left(\frac{f}{B}\right)\left(\frac{f}{r_{o}}\right) \lambda\end{array}$ & $\begin{array}{l}\text { - Maximum tracking veloc- } \\
\text { ity }=2.6 \mathrm{~mm} / \mathrm{s} \\
- \text { Maximum velocity drifts } \\
=1.7 \mathrm{~mm} / \mathrm{s} \text { for } 1 \mathrm{~ms} \text { exposure } \\
\text { time } \\
- \text { Focus tolerance }= \pm 0.6 \mathrm{~mm}\end{array}$ \\
\hline \multirow[t]{4}{*}{$\begin{array}{l}\text { Metrology } \\
\text { gondola }\end{array}$} & \multirow{4}{*}{$\begin{array}{l}\text { Used to align the primary } \\
\text { mirrors: } \\
\text { The Tip-Tilt of each primary } \\
\text { mirror is adjusted by super- } \\
\text { imposing all the return im- } \\
\text { ages of a source at the cur- } \\
\text { vature center, while the pis- } \\
\text { ton is adjusted by searching } \\
\text { the white fringe. } \\
\text { In practice, the source and the } \\
\text { metrology CCD are placed } \\
\text { on the ground and the metrol- } \\
\text { ogy gondola is made of a } \\
\text { convex mirror attached at the } \\
\text { bottom of a girder (Fig. } 8 \text { ). }\end{array}$} & \multirow{4}{*}{$\begin{array}{l}\text { - The top of the girder gon- } \\
\text { dola }(\Omega) \text { must be stable, in a } \\
\text { such a way the return beam } \\
\text { goes on the field mirror at the } \\
\text { ground (Figs. } 3,4 \text { ). } \\
\text { - The altitude of the girder } \\
\text { gondola must be accurate to } \\
\text { focalize the metrology source } \\
\text { near the center of the diluted } \\
\text { primary mirror (also on the } \\
\text { field mirror). }\end{array}$} & \multirow[t]{2}{*}{$\begin{array}{l}- \text { The maximum tolerable } \\
\text { displacement ("horizontaly") } \\
\text { of } \Omega \text { is }\end{array}$} & $\begin{array}{l}-\Delta_{\Omega(x, y)}= \pm 2 \mathrm{~mm} \\
-\Delta_{z}= \pm 2.5 \mathrm{~mm}\end{array}$ \\
\hline & & & & $\begin{array}{ll}\text { with } r_{o} & =100 \mathrm{~mm}, \\
r_{\text {field mirror }} & =300 \mathrm{~mm},\end{array}$ \\
\hline & & & & $R \quad=71.2 \mathrm{~m}$ and \\
\hline & & & $\begin{array}{l}\text { - The position tolerance of } \\
\text { the metrology gondola in } \\
\text { altitude (vertical } \\
\text { displacement) is: } \\
\Delta_{z}= \pm \frac{\lambda}{B} \frac{R^{2}}{r_{0}}\end{array}$ & \\
\hline $\begin{array}{l}\text { Holding } \\
\text { system }\end{array}$ & $\begin{array}{l}\text { The Holding system carries } \\
\text { the metrology gondola, and } \\
\text { the focal gondola above the } \\
\text { primary mirrors. }\end{array}$ & $\begin{array}{l}\text { It can be made of cables un- } \\
\text { der an helium balloon, or ca- } \\
\text { bles attached between moun- } \\
\text { tains, pylons, etc. }\end{array}$ & $\begin{array}{l}\text { - At least at the altitude of } \\
\text { the curvature radius } R \text { of the } \\
\text { primary mirror above the pri- } \\
\text { mary segments. } \\
\text { - It uses material with high } \\
\text { tensile strength and Young's } \\
\text { modulus: Kevelar, Carbon, } \\
\text { etc. } \\
\text { - The holding system can be } \\
\text { stabilized. }\end{array}$ & $\begin{array}{l}\text { - At OHP, a helium balloon } \\
\text { at } 120 \mathrm{~m} \text { altitude tightens a } \\
\text { tripod of PBO cables. } \\
- \text { The top of this stabilized } \\
\text { tripod is at } \approx 71 \mathrm{~m} \text { above the } \\
\text { ground. }\end{array}$ \\
\hline
\end{tabular}

Notes. $R$ is the curvature radius and $f=R / 2$ the focal length of the diluted primary mirror; $r_{\text {field mirror }}$ is the radius of the field mirror; $f_{\text {metrology }}$ the focal length of the metrology mirror; $r_{0}$, the atmospheric Fried parameter ( $\approx 100 \mathrm{~mm}$ at OHP); B the baseline; the wavelength $\lambda=0.5 \mu \mathrm{m}$.

or linked by delay lines have to be pursued. If delay lines are used, the light beams will have to exit the telescopes with a maximum of two to three reflections using, for example, an "Alt-Alt" mount. To minimize the absorption due to many reflections on numerous mirrors, the light could be transported in single mode fibers (Perrin 2004). More studies are required for determining the characteristics of the atmospheric turbulence in valleys compatible with such projects: located relatively high up in the mountains, in a valley oriented in the E-W direction, and with a nearly hemicylindrical shape (Appendix E). If no natural valleys with good seeing conditions can be found, other solutions using pylons could be explored.

Acknowledgements. This research has been funded by CNRS/INSU and Collège de France. Mechanical elements were built by the technical group at OHP and Nice observatory. We thank Chris Bee for improving the English. We are grateful to Jean Surdej, Michel Lintz, and Luc Arnold for some useful corrections and suggestions. We are grateful to student and to the people who helped us during the long nights of tests: Romain Pascal, Jean-Philippe Orts, and Julien Chombart. We are grateful to the LEUKOS company that helped us to use their supercontinuum laser. Google earth and IGN have been used to find sites adapted to a spherical diluted telescope.

\section{Appendix A: Carlina specifications}

In this appendix, the specifications of each part (primary mirror, focal gondola, metrology gondola and holding system) are provided (Table A.1).

\section{Appendix B: The Kevlar cable response}

In this appendix, we briefly describe the approach for selecting the frequency of the servo loop to stabilize the metrology gondola. At this stage in characterizing the system, we focused on the response time of the kevlar cables. We horizontally hung a $100 \mathrm{~m}$ kevlar cable at about $1 \mathrm{~m}$ above the ground. At one end, the cable passes through a pulley and is attached to $17 \mathrm{~kg}$ mass, exerting therefore a tension on the cable that is similar to that of the tripod of the prototype. The other end of the cable is attached to a motorized winch. We used a linear position sensor to detect the mass displacement.

Figure B.1 shows the open-loop step response of the system to a unit movement. The rising time (time delay between the activation of the motorized winch and the detection of the mass motion) is $\approx 50 \mathrm{~ms}$. This time interval constitutes the lower limit 


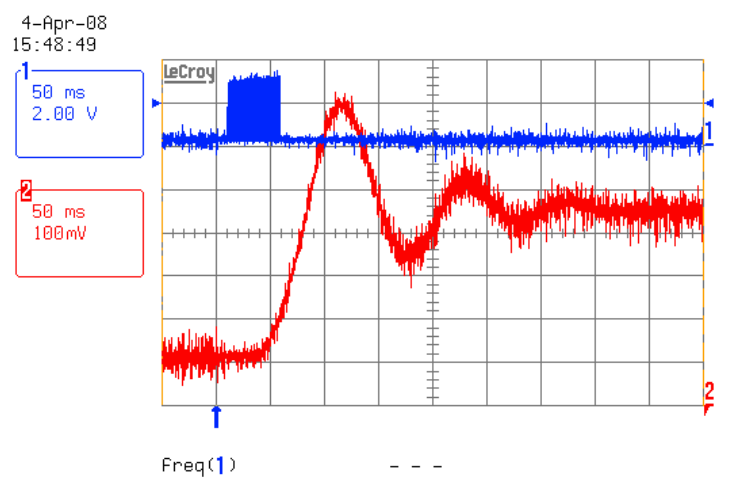

$50 \mathrm{~ms} \quad$ BWL

$1.2 \vee \mathrm{DC} \times$

$210 \mathrm{mV}$ DC ${ }_{10}^{10}$

$45 \mathrm{~V} \times-5 \quad 1 \mathrm{DC} 1.96 \mathrm{~V}$ $500 \mathrm{kS} / \mathrm{s}$
SLOW TRIGGER

Fig. B.1. Oscilloscope window: open-loop step response of a single tensioned Kevlar cable. The blue curve shows the speed of the motorized winch, actuated for $50 \mathrm{~ms}$ (horizontal scale is $50 \mathrm{~ms} / \mathrm{square}$ ). The red curve shows the observed position of the weight versus time. The rise time of this experimental setup is $\approx 50 \mathrm{~ms}$, followed by three dumped oscillations. Settling time is $300 \mathrm{~ms}$.

on the time reaction of the servo loop. In such circumstances, a reasonable period for the operation of the loop could be selected as twice the lower limit imposed by the cable reaction. We therefore set the loop frequency to $10 \mathrm{~Hz}$. The pseudo period and the settling time observed here (Fig. B.1) are not significant, because they mainly depend on the mass and on the geometry of the system. Indeed, $17 \mathrm{~kg}$ is the tension created by the balloon in the cables, but the mass of the metrology gondola is much lighter $\approx 3 \mathrm{~kg}$. This experiment was only destined to evaluate the response time and not the resonant and damping frequency. The frequency of $10 \mathrm{~Hz}$ will be optimized by better characterizing the response of the full system (cable tripod with metrology and focal gondolas) with the PBO cables rather than the response of a single tensioned Kevlar cable (see also discussion Sect. 5.1.1). The rising time of the PBO cables could also be faster than Kevlar because their Young modulus is four times higher.

\section{Appendix C: Experiment log}

Each night of test represents about $20 \mathrm{~h}$ of work to install the balloon, cables, etc. We divided the alignments in four main tasks. Each task has been completed in one to three nights (see Table C.1). We always worked in a low wind $(<20 \mathrm{~km} / \mathrm{h}$ measured with an anemometer attached to the helium balloon). Above $20 \mathrm{~km} / \mathrm{h}$ windspeed, the experiment went down (a cable of the tripod is slackened), and it was not possible to work even with a servo loop.

All the data were obtained on February 23, 2011. We recorded 1596 images of fringes distributed over four hours. We present in this paper the data extracted from 380 images obtain during $15 \mathrm{~min}$ in a coherent block (without touching anything).

\section{Appendix D: System calibration}

The displacements of the photocenters (image of the corner cube) on both PSDs are linked to the length variation of the
Table C.1. Log of the main runs.

\begin{tabular}{lll}
\hline \hline Tasks & Dates & Sect. \\
\hline $\begin{array}{l}\text { Alignment of the metrology } \\
\text { gondola (cables length adjust- } \\
\text { ment, test of the vertical green } \\
\text { laser, etc.) }\end{array}$ & $\begin{array}{l}27 / 07 / 2009 \\
7 / 02 / 2010\end{array}$ & 5.1 \\
\hline $\begin{array}{l}\text { Servo loop (interaction matrix } \\
\text { creation, PID parameters, bug } \\
\text { corrections, etc.) }\end{array}$ & $\begin{array}{l}08 / 03 / 2010 \\
14 / 04 / 2010\end{array}$ & 5.1 .1 and D \\
\hline $\begin{array}{l}\text { Primary mirrors alignment } \\
\text { and fringes detection }\end{array}$ & $\begin{array}{l}26 / 07 / 2010 \\
09 / 2010\end{array}$ & 5.1 .2 and 5.1.3 \\
\hline $\begin{array}{l}\text { Characterization of the system } \\
\text { stability; fringe image record- } \\
\text { ing (Figs. 12 and 13) }\end{array}$ & $23 / 02 / 2011$ & 6 and 6.1 \\
\hline
\end{tabular}

Notes. The dates correspond to the beginning of the night.

tripod cables by the linear equation:

$\Delta \boldsymbol{P}=\left(\begin{array}{c}\Delta P_{x 1} \\ \Delta P_{y 1} \\ \Delta P_{x 2} \\ \Delta p_{y 2}\end{array}\right)=A \cdot \Delta \boldsymbol{L}=\left(\begin{array}{lll}x_{1}^{\prime} & x_{1}^{\prime \prime} & x_{1}^{\prime \prime \prime} \\ y_{1}^{\prime} & y_{1}^{\prime \prime} & y_{1}^{\prime \prime \prime} \\ x_{2}^{\prime} & x_{2}^{\prime \prime} & x_{2}^{\prime \prime \prime} \\ y_{2}^{\prime} & y_{2}^{\prime \prime} & y_{2}^{\prime \prime \prime}\end{array}\right)\left(\begin{array}{l}\Delta L_{1} \\ \Delta L_{2} \\ \Delta L_{3}\end{array}\right)$,

where $\Delta \boldsymbol{P}=\boldsymbol{P}_{\text {measured }}-\boldsymbol{P}_{\text {reference }} ;\left(\Delta P_{x 1}, \Delta P_{y 1}\right)$ and $\left(\Delta P_{x 2}, \Delta P_{y 2}\right)$ are the positions of the photocenters with respect to a reference point (noted $\boldsymbol{P}_{\text {reference }}$ ), respectively on PSD1, and PSD2. $\Delta L_{j}$ is the length variation of the tripod cable (Fig. 8); $A$ is an interaction matrix determined using the following process: ideally, the system must be calibrated during a night without wind and oscillations of the tripod. In practice, the servo loop works well even if the interaction matrix has been created during a slightly windy night; the system returns the girder gondola, in a stepwise linear fashion toward a reference point. In order to determine the interaction matrix values, we actuate successively each motorized winch.

The MW1 motorized winch (Fig. 2) unwinds the cable by a unit value $\Delta L_{1}$ without moving $M W 2$ and $M W 3$. Then, the coordinates of the photocenters on each PSD gives a vector $\left(\Delta P_{x 1}\right.$, $\left.\Delta P_{y 1}, \Delta P_{x 2}, \Delta P_{y 2}\right)$ equal to the first column of $A$, the interaction matrix by replacing $\Delta L_{1}=1, \Delta L_{2}=\Delta L_{3}=0$ in Eq. (D.1):

$$
\left(\begin{array}{lll}
x_{1}^{\prime} & x_{1}^{\prime \prime} & x_{1}^{\prime \prime \prime} \\
y_{1}^{\prime} & y_{1}^{\prime \prime} & y_{1}^{\prime \prime \prime} \\
x_{2}^{\prime} & x_{2}^{\prime \prime} & x_{2}^{\prime \prime \prime} \\
y_{2}^{\prime} & y_{2}^{\prime \prime} & y_{2}^{\prime \prime \prime}
\end{array}\right)\left(\begin{array}{l}
1 \\
0 \\
0
\end{array}\right)=\left(\begin{array}{l}
x_{1}^{\prime} \\
y_{1}^{\prime} \\
x_{2}^{\prime} \\
y_{2}^{\prime}
\end{array}\right)=\left(\begin{array}{l}
\Delta P_{x 1} \\
\Delta P_{y 1} \\
\Delta P_{x 2} \\
\Delta p_{y 2}
\end{array}\right)_{\text {measured by MW1 }} \text {. }
$$

The other columns of the interaction matrix are found by actuating the second and third motor winches:

$$
\left(\begin{array}{lll}
x_{1}^{\prime} & x_{1}^{\prime \prime} & x_{1}^{\prime \prime \prime} \\
y_{1}^{\prime} & y_{1}^{\prime \prime} & y_{1}^{\prime \prime \prime} \\
x_{2}^{\prime} & x_{2}^{\prime \prime} & x_{2}^{\prime \prime \prime} \\
y_{2}^{\prime \prime} & y_{2}^{\prime \prime} & y_{2}^{\prime \prime \prime}
\end{array}\right)\left(\begin{array}{l}
0 \\
1 \\
0
\end{array}\right)=\left(\begin{array}{l}
x_{1}^{\prime \prime} \\
y_{1}^{\prime \prime} \\
x_{2}^{\prime \prime} \\
y_{2}^{\prime \prime}
\end{array}\right)=\left(\begin{array}{l}
\Delta P_{x 1} \\
\Delta P_{y 1} \\
\Delta P_{x 2} \\
\Delta p_{y 2}
\end{array}\right)_{\text {measured by MW2 }}
$$

and

$\left(\begin{array}{lll}x_{1}^{\prime} & x_{1}^{\prime \prime} & x_{1}^{\prime \prime \prime} \\ y_{1}^{\prime} & y_{1}^{\prime \prime} & y_{1}^{\prime \prime \prime} \\ x_{2}^{\prime} & x_{2}^{\prime \prime} & x_{2}^{\prime \prime \prime} \\ y_{2}^{\prime} & y_{2}^{\prime \prime} & y_{2}^{\prime \prime \prime}\end{array}\right)\left(\begin{array}{l}0 \\ 0 \\ 1\end{array}\right)=\left(\begin{array}{l}x_{1}^{\prime \prime \prime} \\ y_{1}^{\prime \prime \prime} \\ x_{2}^{\prime \prime \prime} \\ y_{2}^{\prime \prime \prime}\end{array}\right)=\left(\begin{array}{l}\Delta P_{x 1} \\ \Delta P_{y 1} \\ \Delta P_{x 2} \\ \Delta p_{y 2}\end{array}\right)_{\text {measured by MW3 }}$.

The inverse problem must be solved to determine how much each motor has to move to return the girder gondola to its initial position: $\Delta \boldsymbol{L}=M \cdot \Delta \boldsymbol{P}$. 


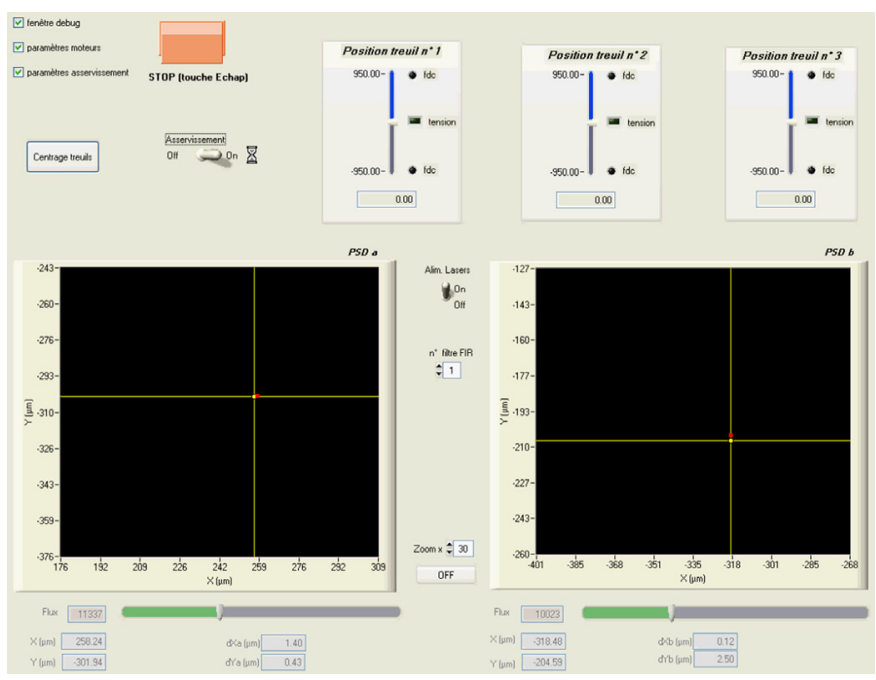

Fig. D.1. Control window of the servo loop. The horizontal green bar shows the flux on the PSDs (it becomes red if the flux is too low). The red point is the position of the photocenter on the PSDa (NE) and PSDb (NW). The yellow cross is the reference point: the servo loop brings back the red point toward this position. Then, the metrology gondola is stabilized around a fixed position. The scale is in microns on the PSD. On these zooms each PSD has a field of view of $18 \mathrm{~mm}$ at the metrology gondola level. Other technical windows used to adjust the PID $\left(K_{\mathrm{p}}, K_{\mathrm{I}}\right.$, $K_{\mathrm{d}}$ ) parameters or to create a matrix of interaction and the reference point are not displayed here. The "position treuil" (motorized winches positions) at the top of the window are used only for manual control.

The inverse $A$ matrix is calculated by using the Singular Value Decomposition (SVD) method for the rectangular matrices (Jacubowiez et al. 1997). The matrix $M$ is computed using the Matlab software SVD function. Finally, a field programmable gate array (FPGA) computes $\Delta \boldsymbol{L}$ in real time:

$\left(\begin{array}{l}\Delta L_{1} \\ \Delta L_{2} \\ \Delta L_{3}\end{array}\right)=\left(\begin{array}{llll}m_{11} & m_{12} & m_{13} & m_{14} \\ m_{21} & m_{22} & m_{23} & m_{24} \\ m_{31} & m_{32} & m_{33} & m_{34}\end{array}\right)\left(\begin{array}{l}\Delta P_{x 1} \\ \Delta P_{y 1} \\ \Delta P_{x 2} \\ \Delta P_{y 2}\end{array}\right)$.

The servo loop system is piloted via a graphical interface (Fig. D.1).

\section{Appendix E: Discussion}

\section{E.1. Diluted telescopes}

The science targets will dictate the density of mirrors required in the pupil of the future interferometers because the typical step of the diluted pattern imposes the "Crowding Field of View" (FOV) limits (Lardière et al. 2007; Koechlin 2003): if the interferometer is very diluted (long distance between the subapertures), images of large extended objects (angularly on the sky) reaches the crowding limit (Lardière et al. 2007), and is not observable. This limitation has already been reached on the bigger stars with existing interferometers. For instance, some Miras are not easily observable with long baseline interferometers such as CHARA, Keck, or the VLTI. For example, to obtain a direct image of a 30 mas object with a $100 \mathrm{~m}$ aperture interferometer, at least 30 mirrors are required in the pupil. Finally, to obtain a very high angular resolution (submilliarcsec), and many "resels" in the image, future interferometers (post-VLTI) will require tens or hundreds of subapertures (rich uv coverage) over an area of $\approx 100-300 \mathrm{~m}$ in diameter, and a few telescopes distributed on kilometer baselines (for very high angular resolution). For the same reasons, the ALMA submillimeter interferometer project consists of a giant array of $12 \mathrm{~m}$ antennas with baselines of up to $16 \mathrm{~km}$, and an additional compact array of 7-12 $\mathrm{m}$ antennas to enhance its ability for imaging extended sources (Tarenghi 2008).

The Post-VLTIs will then be complex systems that will work with many mirrors, actuators, and servo loops; they will be equipped with adaptive optics and cophasing devices; their imaging properties will resemble those of ELT telescopes but they will provide much higher angular resolution. Their wavefront sensors will probably be similar to the fringe tracking sensors adapted to the diluted pupils (Borkowski et al. 2005; Tarmoul et al. 2010). To build these diluted telescopes made of hundreds of subapertures, the combined knowledge of the scientific communities working on ELTs and interferometers will be required.

We think that Carlina is a part of this new family of instruments dedicated to unravel at high angular resolution the Universe: The diluted telescopes. The MMT (Carleton 1979) and the large binocular telescope interferometer (LBT) with its two $8.4 \mathrm{~m}$ primaries, and its $23 \mathrm{~m}$ optical baseline could be precursors of these Diluted Telescopes (Hinz 2001; Kim 2010).

In order to achieve high imaging capabilities, and to be more sensitive than present interferometers, a diluted telescope will require the following characteristics:

- A relatively dense array of mirrors (rich uv coverage).

- The optical train will be simplified to minimize the reflections between the primary mirrors and the focal instrument (probably without delay lines).

- Focal instruments will be optimized to be sensitive and adapted to the science goals: AO adapted to the diluted telescopes, and coronagraphy will be implemented. The way to combine the telescopes will have to be optimized (see discussions in Patru et al. 2008; Menut et al. 2008).

From this point of view, Carlina, which works without delay lines, is an ideal solution to recombine many mirrors. As a next step, we shall propose to build a $100 \mathrm{~m}$ aperture diluted telescope that we will call a LDT or very large diluted telescope (VLDT) respectively for an equivalent surface of a few meters square or $50 \mathrm{~m}^{2}$ (VLT surface).

A (V)LDT could be recombined in the densified mode (Tallon 1992; Labeyrie 1996), or using any other technique depending on the science goals (more studies are required).

It is also possible that the post-ELTs will be partially diluted during their early construction phase. It will then be also feasible to recombine the future ELTs, with auxiliary telescopes on kilometric baselines, using single-mode fibers (Perrin et al. 2000).

\section{E.2. The site}

The constraints for a (V)LDT's site are the same as for any modern telescope: it should be located relatively high in the mountains (dry air for IR observations), in a good weather area (weak cloud cover) and with a favorable atmospheric turbulence ( seeing $<1$ ", slow turbulence). The "Seeing" of the valleys will have to be studied in detail as it was done for example at Paranal (Dali et al. 2010). Nevertheless, the selection relies mainly on topographic considerations. A valley oriented in the $\mathrm{E}-\mathrm{W}$ direction is required, with a nearly hemicylindrical shape at the bottom (Fig. E.1). 
Table E.1. Main characteristics of sites found in the southern Alps.

\begin{tabular}{|c|c|c|c|c|c|}
\hline Sites name & Coordinates & $\begin{array}{l}\text { Valley } \\
\text { bottom } \\
\text { altitude }\end{array}$ & $\begin{array}{l}\text { Valley } \\
\text { top altitude } \\
\text { N-S sides }\end{array}$ & $\begin{array}{l}\text { Curvature } \\
\text { radius } \\
( \pm 10 \mathrm{~m})\end{array}$ & $\begin{array}{l}\text { Baseline } \\
\text { at } F / 2\end{array}$ \\
\hline La Moutière & $\begin{array}{l}44^{\circ} 19^{\prime} \mathrm{N} \\
6^{\circ} 46^{\prime} \mathrm{E}\end{array}$ & $2070 \mathrm{~m}$ & $\begin{array}{l}2700 \mathrm{~m} \text { at north } \\
2600 \mathrm{~m} \text { at south }\end{array}$ & - & - \\
\hline Estrop & $\begin{array}{l}44^{\circ} 16^{\prime} \mathrm{N} \\
6^{\circ} 31^{\prime} \mathrm{E}\end{array}$ & $2040 \mathrm{~m}$ & $\begin{array}{l}2720 \mathrm{~m} \\
2460 \mathrm{~m}\end{array}$ & $600 \mathrm{~m}$ & $150 \mathrm{~m}$ \\
\hline Meollion & $\begin{array}{l}44^{\circ} 43^{\prime} \mathrm{N} \\
6^{\circ} 17^{\prime} \mathrm{E}\end{array}$ & $1670 \mathrm{~m}$ & $\begin{array}{l}2800 \mathrm{~m} \\
2380 \mathrm{~m}\end{array}$ & - & - \\
\hline Chaumeille & $\begin{array}{l}44^{\circ} 45^{\prime} \mathrm{N} \\
6^{\circ} 18^{\prime} \mathrm{E}\end{array}$ & $1580 \mathrm{~m}$ & $\begin{array}{l}2670 \mathrm{~m} \\
2830 \mathrm{~m}\end{array}$ & - & - \\
\hline Le Casset & $\begin{array}{l}44^{\circ} 49^{\prime} \mathrm{N} \\
6^{\circ} 13^{\prime} \mathrm{E}\end{array}$ & $1150 \mathrm{~m}$ & $\begin{array}{l}2960 \mathrm{~m} \\
2300 \mathrm{~m}\end{array}$ & - & - \\
\hline Freissinières & $\begin{array}{l}44^{\circ} 44^{\prime} \mathrm{N} \\
6^{\circ} 28^{\prime} \mathrm{E}\end{array}$ & $1370 \mathrm{~m}$ & $\begin{array}{l}3000 \mathrm{~m} \\
2450 \mathrm{~m}\end{array}$ & $450 \mathrm{~m}$ & $112.5 \mathrm{~m}$ \\
\hline Deslioures & $\begin{array}{l}44^{\circ} 47^{\prime} \mathrm{N} \\
6^{\circ} 27^{\prime} \mathrm{E}\end{array}$ & $1560 \mathrm{~m}$ & $\begin{array}{l}2700 \mathrm{~m} \\
3000 \mathrm{~m}\end{array}$ & $400 \mathrm{~m}$ & $100 \mathrm{~m}$ \\
\hline Le Villard & $\begin{array}{l}44^{\circ} 50^{\prime} \mathrm{N} \\
6^{\circ} 26^{\prime} \mathrm{E}\end{array}$ & $1270 \mathrm{~m}$ & $\begin{array}{l}2570 \mathrm{~m} \\
2450 \mathrm{~m}\end{array}$ & $600 \mathrm{~m}$ & $150 \mathrm{~m}$ \\
\hline Les Etages & $\begin{array}{l}44^{\circ} 56^{\prime} \mathrm{N} \\
6^{\circ} 16^{\prime} \mathrm{E}\end{array}$ & $1640 \mathrm{~m}$ & $\begin{array}{l}3200 \mathrm{~m} \\
2870 \mathrm{~m}\end{array}$ & & - \\
\hline
\end{tabular}

Notes. A minus sign is used in the boxes where we could not determine a value due to a lack of numerical ground data. But, typically, all these valleys have about the same orientations $(\mathrm{E} / \mathrm{W})$, size, and shape.

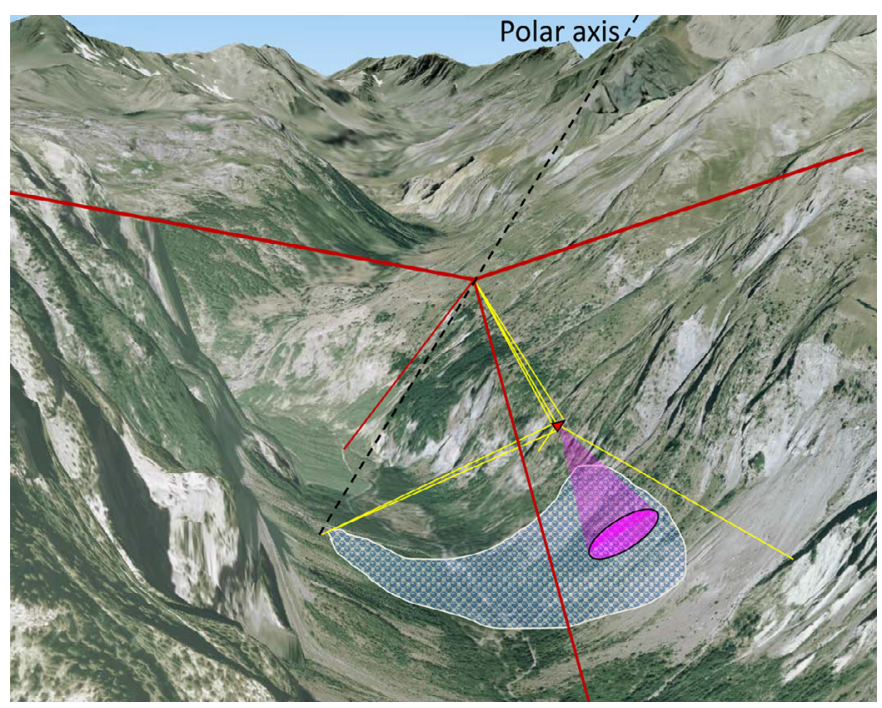

Fig. E.1. Drawing of a diluted telescope in the Valley. The pink circle represents the effective aperture of $120 \mathrm{~m}$ at $F / 2$.

The price tag of the project will mainly depend on the site chosen to build a (V)LDT. For this reason, we distinguish between two kinds of site:

- The extremely good areas (ex: Paranal site) where we could propose only in a second stage to build a VLDT (100-300 m aperture) perhaps surrounded by telescopes over kilometric baselines.

- The "intermediate" sites: the weather and turbulence are acceptable but generally not ideal; it is relatively easy to access the sites by road and to connect them to power, internet, etc. The focal instrument will be optimized for such a turbulence.

We consider that the construction of a scientific demonstrator of LDT with $\mathrm{a} \approx 100 \mathrm{~m}$ aperture is worth considering within the next ten years. More studies are required to determine whether some intermediate sites are really adapted to build an LDT for a reasonable cost. For a focal gondola at $f / 2$ and an effective aperture of $100 \mathrm{~m}$, valleys of 400-600 m of curvature radius are required. By using Google Earth, and the "Institut Géographique National" (IGN) maps, we found in the southern Alps, nine intermediate sites that could have an adapted shape for a future LDT. Their preliminary characteristics are given in Table E.1.

Detailed studies are necessary to determine precisely the properties (geometry, atmospheric seeing, snow and rock avalanches, etc.) of potential sites. A study using a total station or/and aerial stereoscopic pictures will have to be done on the best sites. This study should determine the height of the primary mirror mounts in order to have a relatively homogeneous coverage of mirrors in the valley. A study of the stability of the mounts in function of their heights, will be required.

For example, a drawing of the installation of a diluted telescope in Freissinières valley is presented in Fig. E.1. In such a site, it should be possible to track any star that passes above $40^{\circ}$ from the horizon at meridian during about two hours. Geometrically, Freissinières is a good site but it is surrounded by waterfalls, screes, etc.

Another possibility that should be explored is to put the primary segments at the top of pylons (Fig. E.2) in order to build a (V)LDT in a site with extremely good conditions of atmospheric turbulence without topographic constraints. In this case, the 10 $200 \mathrm{~m}$ pylons could be stabilized by a device equivalent to the system used for the metrology gondola described in this paper. The thermal expansion of a $100 \mathrm{~m}$ steel pylon would be around $1.2 \mathrm{~cm}$ for $10^{\circ} \mathrm{C}$ variation. We can then expect a drift of about $\approx 1 \mu \mathrm{m} / \mathrm{s}$ during the night. A second servo loop stage, at the top of each pylon would therefore be necessary to control the piston and tip-tilt of each mirror. Even though this might a priori appear to complicate the design, one should keep in mind the comparison with systems including tens of delay lines: it is probably easier to stabilize a mirror around a fixed position than to control a delay line moving at several millimeters per second. Moreover, the internal metrology at the curvature center allows the coherencing of the primary mirrors without any stellar source. 


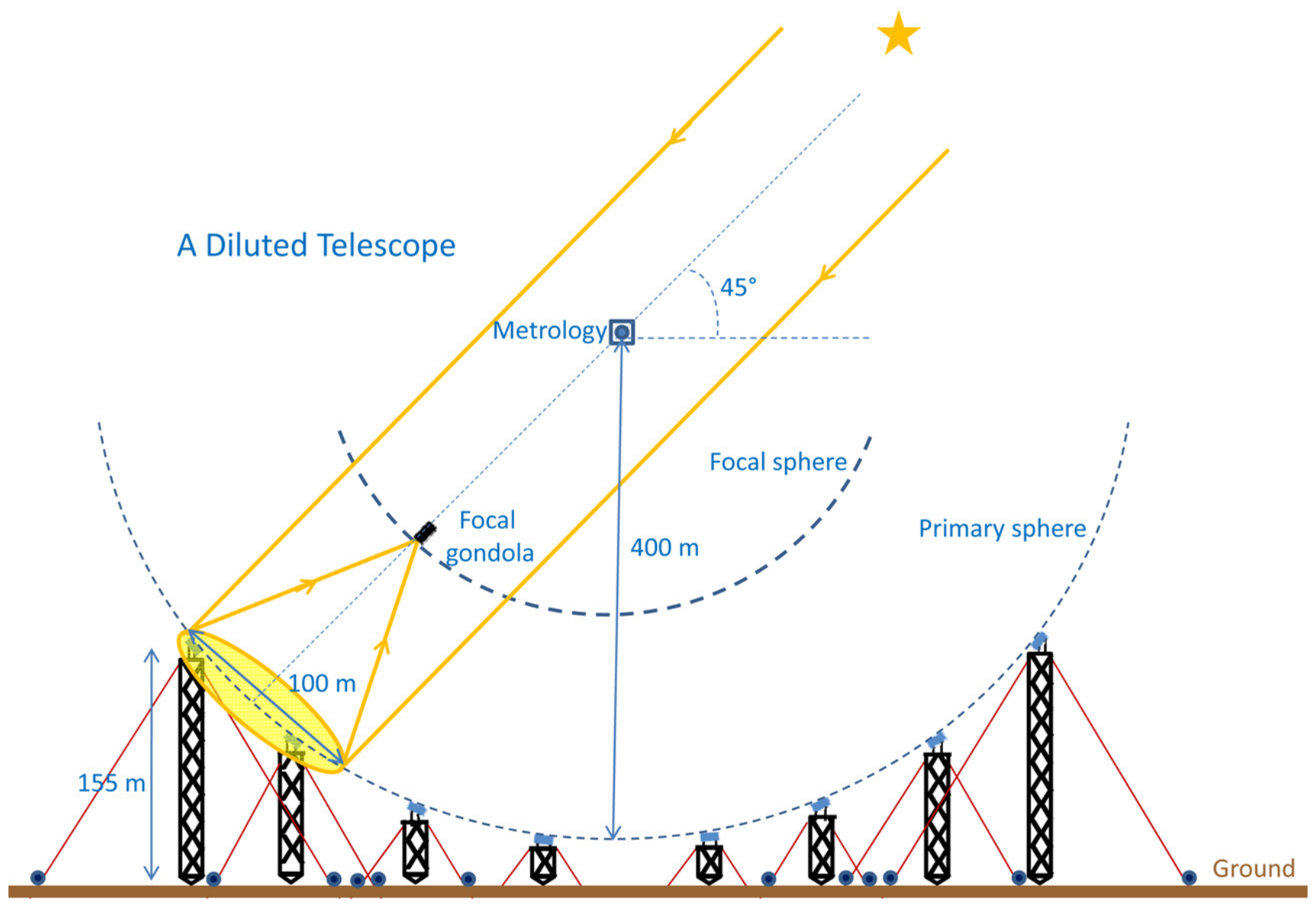

Fig. E.2. Schematic view of a Diluted Telescope using pylons to support the primary mirrors. The red cables are motorized to stabilize the top of the pylons using the same kind of servo loop system as that described in Sect. 5.1.1. A second mechanical stage stabilizes the primary mirrors within an accuracy of 1 micron using a metrology at the curvature center as described in this article.

More studies are also required installing a pylon array with many cables (that must not touch each other even in strong wind conditions). The impact on the local turbulence of such a massive structure that gets cold during the night has also to be taken into account. Digging an artificial crater in a flat terrain seems to be impractical.

\section{References}

Blazit, A., Rondeau, X., Thiébaut, É., et al. 2008, ApOpt., 47, 1141 Borkowski, V., Labeyrie, A., Martinache, F., \& Peterson, D. 2005, A\&A, 429, 747

Carleton, N. P. 1979, in The MMT and the Future of Ground-Based Astronomy, Proc. Symp. held to mark the dedication of the Multiple Mirror Telescope at the Mount Hopkins Observatory, Arizona on May 9, ed. C. Trevor, Weekes, SAO Special Report, 385, 37

Courde, C., Lintz, M., \& Brillet, A. 2009, Meas. Sci. Technol., 20, 127002

Courde, C., Lintz, M., \& Brillet, A. 2010, Instrumentation Mesures Métrologie, 10,81

Dali Ali, W., Ziad, A., Berdja, A., et al. 2010, A\&A, 524, A73

De Becker, M., Le Coroller, H., \& Dejonghe, J. 2011, in Proc. of the 39th Liège Astrophysical Colloq., ed. G. Rauw, M. De Becker, Y. Naze, J.-M. Vreux, \& P. M. Williams, BSRSL, 80, 486

D'Odorico, S., Ramsay, S., Hubin, N., et al. 2010, The Messenger, 140, 17 Hinz, P. 2001, in 198th AAS Meeting, BAAS, 33, 859

Jacubowiez, L., Sauer, H., Bernard, F., Plantegenest, M. T., \& Avignon, T. 1997, SupOptique/IOTA, Colloque CETSIS, Orsay, 20-21 Nov.

Kim, J., Hinz, P., Durney, O., et al. 2010, in Optical and Infrared Interferometry II, Proc. SPIE, 7734, 77341W

Koechlin, L. 2003, EAS, 8, 349
Labeyrie, A. 1996, A\&AS, 118, 517

Lardière, O., Labeyrie, A., Mourard, D., et al. 2003, in Interferometry for Optical Astronomy II, ed. W. A. Traub, Proc. SPIE, 4838, 1018

Lardière, O., Martinache, F., \& Patru, F. 2007, MNRAS, 375, 977

Le Coroller, H., Dejonghe, J., Arpesella, C., Vernet, D., \& Labeyrie, A. 2004, A\&A, 426, 721 (Paper I)

Le Coroller, H., Dejonghe, J., Regal, X., et al. 2010, in Proceedings of JENAM 2010, Lisbon 6-10 September 2010, ed. A. Moitinho, E. Amores, V. Arsenijevic, J. Ascenso, \& R. Azevedo, 95

Meisenheimer, K. 2009, in Science with the VLT in the ELT Era, Astrophysics and Space Science Proceedings (Springer Netherlands), 507

Menut, J. L., Valat, B., Lopez, B., et al. 2008, ApJ, 686, 1514

Mertz, L. 1996, Excursions in Astronomical Optics (New York: Springer-Verlag, Inc.)

Mourard, D., Clausse, J. M., Marcotto, A., et al. 2009, A\&A, 508, 1073

Orndoff, E. 1995, NASA, Lyndon B. Johnson Space Center Houston Texas, Development and Evaluation of Polybenzoxazole Fibrous Structures, Technical Memorandum, 104814

Patru, F., Mourard, D., Clausse, J. M., et al. 2008, A\&A, 477, 345

Patru, F., Tarmoul, N., Mourard, D., \& Lardière, O. 2009, MNRAS, 395, 2363

Perrin, G., Lai, O., Lena, P. J., \& Coudé du Foresto, V. 2000, in Interferometry in Optical Astronomy, Proc. SPIE, 4006, 708

Perrin, G., Lai, O., Woillez, J., et al. 2004, AAS Meeting 204, BAAS, 36, 982

Petrov, R. G., Malbet, F., Weigelt, G., et al. 2007, A\&A, 464, 1

Ridgway, S., \& Glindemann, A. 2010, EAS Publ. Ser., 40, 235

Tallon, M., \& Tallon-Bosc, I. 1992, A\&A, 253, 641

Tarenghi, M. 2008, Astrophys Space Sci., 313, 1

Tarmoul, N., Mourard, D., Hénault, F., et al. 2010, SPIE Conf., 7734, 68

Weigelt, G., Mourard, D., Abe, L., et al. 2000, Interferometry in Optical Astronomy, ed. P. J. Lena, \& A. Quirrenbach, Proc. SPIE, 4006, 617

Woillez, J., Perrin, G., \& Lai, O. 2005, BAAS, 37, 1308 